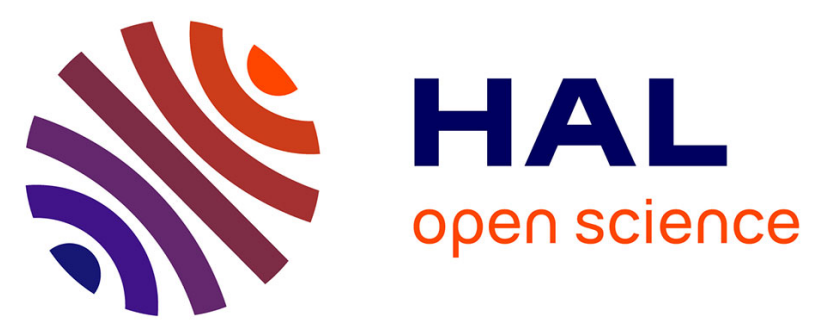

\title{
Analysis of the Magnetic Exchange Interactions in Yttrium(III) Complexes Containing Nitronyl Nitroxide Radicals
}

Julie Jung, Marin Puget, Olivier Cador, Kevin Bernot, Carmen J. Calzado, Boris Le Guennic

\section{To cite this version:}

Julie Jung, Marin Puget, Olivier Cador, Kevin Bernot, Carmen J. Calzado, et al.. Analysis of the Magnetic Exchange Interactions in Yttrium(III) Complexes Containing Nitronyl Nitroxide Radicals. Inorganic Chemistry, 2017, 56 (12), pp.6788-6801. 10.1021/acs.inorgchem.6b02952 • hal-01544247

HAL Id: hal-01544247

https://hal-univ-rennes1.archives-ouvertes.fr/hal-01544247

Submitted on 13 Sep 2017

HAL is a multi-disciplinary open access archive for the deposit and dissemination of scientific research documents, whether they are published or not. The documents may come from teaching and research institutions in France or abroad, or from public or private research centers.
L'archive ouverte pluridisciplinaire HAL, est destinée au dépôt et à la diffusion de documents scientifiques de niveau recherche, publiés ou non, émanant des établissements d'enseignement et de recherche français ou étrangers, des laboratoires publics ou privés. 


\title{
Analysis of the Magnetic Exchange
}

\section{Interactions in Yttrium(III) Complexes}

\section{Containing Nitronyl Nitroxide Radicals}

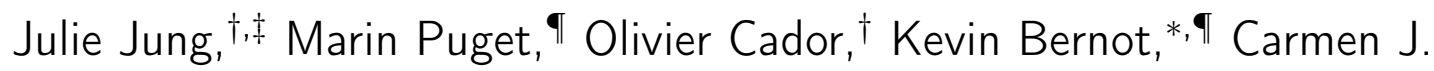
Calzado, ${ }^{*, \S}$ and Boris Le Guennic ${ }^{*, \dagger}$

Institut des Sciences Chimiques de Rennes, UMR 6226 CNRS, Université de Rennes 1, 263 Avenue du Général Leclerc, 35042 Cedex Rennes, France, Max Planck Institut für Chemische Energiekonversion, Stiftstr. 34-36, D-45470 Mülheim an der Ruhr, Germany, INSA, Institut des Sciences Chimiques de Rennes UMR CNRS 6226, 35708 Rennes, France, and Departamento de Química Física. Universidad de Sevilla. c/ Prof. García González, s/n. 41012 Sevilla, Spain

E-mail: kevin.bernot@insa-rennes.fr; calzado@us.es; boris.leguennic@univ-rennes1.fr

\begin{abstract}
We report a combined theoretical and experimental investigation of the exchange interactions governing the magnetic behavior of a series of nitronyl-nitroxide (NIT)based Y(III) complexes, i.e. $\mathrm{Y}(\mathrm{hfac})_{3}(\mathrm{NIT}-\mathrm{R})_{2}$ with $\mathrm{R}=\mathrm{PhOPh}(\mathbf{1})$, PhOEt (2) and PhOMe (3a, 3b). Even if some of these complexes or their Dy(III) parents were already described in the literature [Zhao et al. Trans. Met. Chem. 2006, 31, 593; Bernot et

\footnotetext{
*To whom correspondence should be addressed

${ }^{\dagger}$ Université de Rennes

$\ddagger$ Present address: Max Planck Institut für Chemische Energiekonversion

"INSA de Rennes

$\S$ Universidad de Sevilla
} 
al. J. Am. Chem. Soc. 2009, 131, 5573], their synthesis procedure as well as their structural and magnetic properties were completely reconsidered. Depending on the nature of $\mathrm{R}$ and of crystallization conditions, $\mathrm{Y}(\mathrm{hfac})_{3}(\mathrm{NIT}-\mathrm{R})_{2}$ units can be organized as supramolecular dimers, linear or orthogonal chains. Such structural diversity within the series induces extremely different magnetic behaviors. The observed behaviors are rationalized by state-of-the-art wave-function-based quantum chemical approaches (CASSCF/DDCI) that demonstrate the existence of effective intra-molecular interactions between the NIT-R radical ligands of an isolated $\mathrm{Y}(\mathrm{hfac})_{3}(\mathrm{NIT}-\mathrm{R})_{2}$ molecule, but also between NIT-R moieties belonging to different $\mathrm{Y}(\mathrm{hfac})_{3}(\mathrm{NIT}-\mathrm{R})_{2}$ units. These results are supported by the use of spin Hamiltonian models going beyond the basic Bleaney-Bowers formalism to properly fit the experimental magnetic data. Finally, the microscopic mechanisms behind the evidenced intramolecular exchange interactions are elucidated through the inspection of the calculated wave-function. In particular, whereas the role of Y orbitals was already proposed, we herein demonstrate the contribution of the $\mathrm{hfac}^{-}$ancillary ligands in mediating the magnetic interaction between the NIT radicals. 


\section{Introduction}

Since the early 90's the magnetic behavior of isolated molecules has caught much attention. ${ }^{1}$ The discovery of molecules that present magnetic bistability of molecular origin ${ }^{2}$ made molecular magnetism emerge as an intense field of research. ${ }^{3}$ In particular, Single Molecule Magnets (SMMs) have been evidenced as particularly suitable entities for information storage and processing, ${ }^{4}$ spintronic, $\stackrel{[5}{8}$ or cryogenic ${ }^{9}$ devices.

Basically, SMM behavior is achieved in molecules having both a strongly axial magnetic anisotropy and a high energy barrier to the reversal of their magnetic moment.10111 While the former property guarantees magnetic bistability by making the ground state a doublet and prevents fast relaxation in this doublet ground state by limiting the mixing between the two associated sub-states, the latter establishes the temperature window within which slow relaxation can be observed. Ultimately, the relaxation rate is only determined by the spin-phonon coupling mechanisms involved in the magnetic relaxation, which are completely system-dependent since correlated to the vibrations of both the molecule and the lattice.

The optimization of the performance of SMMs, especially the temperature window where they depict magnetic slow relaxation, can be achieved through different strategies. The first and simplest one consists in synthesizing poly-nuclear complexes ${ }^{12}$ in order to increase the overall anisotropy of the molecule but the correlation between the anisotropy (D) and the total spin $(\mathrm{S})$ of the molecule make this strategy hard to achieve ${ }^{13}$ provided that the respective arrangement of the ions is carefully controlled.14]15 A second approach is to use highly symmetrical and rigid ligands to carefully engineer ligand field and favor stabilization of ground-state magnetic moment along a defined axis. ${ }^{[16}$ This approach is particularly suitable for SMMs that use highly anisotropic lanthanide ions as spin carriers. ${ }^{17} \mathrm{~A}$ third route is based on the use of isotopically-pure metal ion derivatives that avoid spontaneous loss of the magnetic bistability in zero-field. ${ }^{[8 \mid 19}$ Finally, a last strategy relies on the use of radical ligands that can induce strong magnetic exchange between anisotropic spin carriers. ${ }^{20 \mid 21}$ One of the best examples of the latter strategy is given by the $\mathrm{N}_{2}^{3-}$ radical-bridged terbium(III) 
complex by J. R. Long and coworkers, $22 \sqrt[24]{24}$ which remains among the SMMs with the highest working temperature available to date.

Among well-known organic radicals, nitronyl-nitroxides (NIT) are particularly appealing since they allow to obtain edifices of various dimensionalities. In these architectures, significant magnetic coupling can be observed that induces 0-, 1-, 2- or 3-dimensional overall magnetic behaviors. $25+33$ Such materials can also give rise to unique magnetic behavior such as next-nearest neighbor interactions, $\frac{34}{34}$ one-dimensional helimagnetism $35 \sqrt[37]{37}$ or Single Chain Magnets (SCMs) behavior. 138 Among the latter class of compounds, SCMs of formula $\left[\mathrm{Ln}(\mathrm{hfac})_{3}(\mathrm{NIT}-\mathrm{PhOPh})\right]_{\infty}$, where hfac $^{-}=$hexafluoroacetylacetonate and NIT-PhOPh $=2,4$ '-benzoxo-4,4,5,5-tetramethyl-imi-dazoline-1-oxyl-3-oxide, were found to have a quite complex magnetic behavior $\underline{41}$ and deep investigation of their monomeric constitutive units was necessary for its rationalization. $\frac{42}{2}$ From the pioneering work by Benelli, Caneschi and Gatteschi on the $\operatorname{Ln}(\text { hfac })_{3}(\mathrm{NIT}-\mathrm{R})_{2}$ compounds, it appears that the coupling interaction between the lanthanide ion and the radical ligand (Ln-NIT) can be either ferromagnetic (FM) or antiferromagnetic (AFM) depending on the nature of the lanthanide ion while magnetic interaction between the two radical ligands (NIT-NIT) is always found antiferromagnetic. $25[3443$ 48 Rationalization of these exchange interactions has been performed initially using angular overlap analysis. $\frac{25 \mid 46}{2}$ On the basis of $\mathrm{Y}(\mathrm{hfac})_{3}(\mathrm{NIT}-\mathrm{R})_{2}$ investigations, three possible mechanisms for the NIT-NIT interaction have been proposed: (i) direct exchange, (ii) super-exchange through the metal ion and (iii) super-exchange through the non-radical hfac- ligands. $\frac{45]}{47}$ However, definitive validation of the magnetic coupling mechanisms in these relatively simple units is still lacking. This is due, on the one hand, to the peculiar electronic structure of the lanthanide ions that induces great variety of magnetic coupling mechanisms (isotropic and anisotropic exchange, dipolar coupling...) $\frac{49}{4}$ and on the other hand, on the numerous exchange pathways potentially responsible for the overall magnetic behavior.

Fundamentally, magnetic exchange is related to electronic repulsion and more precisely 
to the Fermi hole surrounding the electrons. This interaction is thus mediated through electronic spin. ${ }^{49}$ From the computational point of view, as for magnetic anisotropy or ligand-field effects,,$\frac{50,54}{54}$ procedures to investigate magnetic exchange rely on the use of a model Hamiltonian which is derived either from chemical intuition or using the effective Hamiltonian theory. ${ }^{55156}$ In the simplest case, i.e. two half spins interacting isotropically, magnetic exchange can then be effectively described using the Heisenberg-Dirac-Van Vleck Hamiltonian. Yet, as soon as spin-orbit coupling (SOC) or local anisotropy arises, this Hamiltonian needs to be further refined, 57 which can complicate greatly the physical picture of the system. As a consequence, ab initio calculations can become quite intricate, especially for lanthanidebased complexes, where SOC and magnetic anisotropy are rather strong. The approach is further complicated since lanthanide ions interact rather poorly through magnetic exchange with other spin-carriers such as transition metals or organic radicals, with coupling constants not exceeding $10 \mathrm{~cm}^{-1}$. This is mainly because the $4 \mathrm{f}$ shell of lanthanide trivalent ions is quite efficiently shielded by the outter $5 \mathrm{~s}$ and $5 \mathrm{~d}$ shells. This is the main reason why there are to date very few in-depth theoretical studies of magnetic exchange in lanthanide or more generally rare earth-based complexes. $\cdot 58159$ For all these reasons, and as highlighted by Benelli and coworkers, an alternative approach consists in studying first magnetic exchange in diamagnetic derivatives such as $\mathrm{Y}(\mathrm{III}), \mathrm{La}(\mathrm{III})$ or $\mathrm{Lu}(\mathrm{III})$. Consequently, to disentangle all the relative contributions to magnetic exchange in the $\operatorname{Ln}(\text { hfac })_{3}(\mathrm{NIT}-\mathrm{R})_{2}$ family, the complex $\mathrm{Y}(\mathrm{hfac})_{3}(\mathrm{NIT}-\mathrm{PhOPh})_{2}$ has been chosen as target compound for the present study. ${ }^{42}$

The magnetic behavior is then dominated by intramolecular interactions between the radicals. However, uncoordinated NO groups are known to bear non-negligible electronic density $\underline{602}$ and their respective geometric arrangement and distances in the crystal packing can give rise to sizeable intermolecular magnetic interactions (NIT $\cdots$ NIT interactions) that may compete with the intramolecular (NIT-NIT) ones. We have thus chosen to use different substituents on the aliphatic group of the nitronyl-nitroxide radical to afford structural diversity to our study. Three different substitutions in para position of the phenyl ring have 
been explored affording three derivatives of formula $\mathrm{Y}(\mathrm{hfac})_{3}(\mathrm{NIT}-\mathrm{R})_{2}$ with $\mathrm{R}=\mathrm{PhOPh}(\mathbf{1})$, $\operatorname{PhOEt}(\mathbf{2})^{[4]}$ and PhOMe $\left(\mathbf{3 a},{ }^{[63} \mathbf{3 b}\right)$ (Scheme 1). They present very different supramolecular architectures, from dimers to linear or orthogonal chains, with a wide range of intermolecular distances (from 2.80 to $4.26 \AA$ ). Consequently, our study goes far beyond the investigation of SCM monomer's and a simple description of these systems through HDVV Hamiltonian. In fact, high level $a b$ initio calculations provide a reliable qualitative and quantitative picture of the magnetic behavior. Moreover, insights on the microscopic mechanisms responsible for the through-yttrium magnetic interactions in this series of complexes are provided.

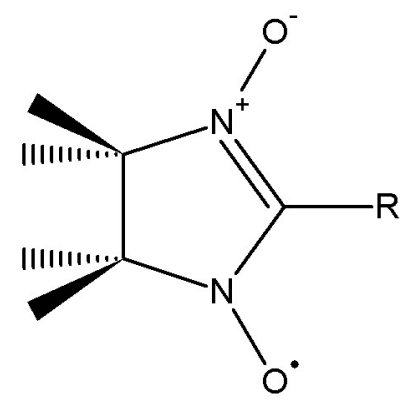

Scheme 1: Schematic representation of the nitronyl-nitroxide radical ligands used. Corresponding complexes of formula $\mathrm{Y}(\mathrm{hfac})_{3}(\mathrm{NIT}-\mathrm{R})_{2}$ for $\mathrm{R}=\mathrm{PhOPh}$, PhOEt and PhOMe are named $\mathbf{1}, \mathbf{2}, \mathbf{3} \mathrm{a}$ and $\mathbf{3 b}$, respectively.

\section{Experimental section}

\section{Synthesis and X-ray Crystallography}

Crystallization procedures for $\mathrm{Y}(\mathrm{hfac})_{3}(\mathrm{NIT}-\mathrm{R})_{2}$ species vary drastically depending of both the nature of the - $\mathrm{R}$ substituent and the crystallization temperature $\left(\mathrm{T}_{\text {crist }}\right)$. For $\mathrm{R}=\mathrm{PhOPh}$, crystals could be obtained only for the Dy derivative (1', CCDC-1519869). Powders of 1 are found isostructural to 1' by comparison with their powder X-ray diffraction patterns (Figure S1). For $\mathrm{R}=$ PhOEt, only one species, 2 , is obtained whatever $\mathrm{T}_{\text {crist. }}{ }^{42}$ For $\mathrm{R}=$ $\mathrm{PhOMe}$, if $\mathrm{T}_{\text {crist }}<5^{\circ} \mathrm{C}$, the already reported compound $\mathbf{3 a}$ is mainly obtained (CCDC178130), ${ }^{63}$ whereas for $\mathrm{T}_{\text {crist }}=20^{\circ} \mathrm{C}$ a majority of crystals of $\mathbf{3 b}$ are found. Scanning of the 
crystallization temperature conditions does not optimize the $\mathbf{3 a} / \mathbf{3} \mathbf{b}$ ratio.

Single crystals of all derivatives were mounted on a APEXII AXS-Bruker diffractometer $(150 \mathrm{~K})$ with $\mathrm{Mo} \mathrm{K} \alpha$ radiation $(\lambda=0.71073 \AA)$. Data reduction and cell refinement were performed with Denzo and Scalepack programs. $\underline{64}$ The crystal structures have been solved by direct methods using SIR97 program, $\underline{65}$ and refined with full matrix least-square methods based on $\mathrm{F}^{2}$ (SHELX97) ${ }^{66}$ with WINGX program. ${ }^{67}$ All non-hydrogen atoms were refined anisotropically using SHELXL program. Hydrogen atoms bound to the organic ligand were located at ideal positions. Final structure refinement data of four derivatives are listed in Tables S1 and S2, Full details of the X-ray structure determination of the four crystal structures have been deposited at the Cambridge Crystallographic Data Center under the depository numbers CCDC-1519220 for $\mathbf{2}$ and CCDC-1519219 for $\mathbf{3 b}$. They can be obtained free of charge at http://www.ccdc.cam.ac.uk/conts/retrieving.html (or from the Cambridge Crystallographic Data Centre, 12, Union Road, Cambridge CB2 IEZ, UK; fax: (internat.) +44-1223/336-033; E mail: deposit@ccdc.cam.ac.uk), on request, from the authors and the reference to this publication.

Powder X-ray diffraction diagram of $\mathbf{1}$ has been collected at room temperature using a PanalyticalX'Pert Pro diffractometer equipped with an X'Celerator detector. Calculated pattern of 1' was produced using Mercury 3.0 program.

\section{Magnetic measurements}

Magnetic measurements were performed on powder pellets with a MPMS Quantum Design magnetometer between 2 and 300K. All measurements have been corrected from sample holder contribution and from diamagnetic contributions as calculated by Pascal's constants. 


\section{Computational details}

\section{$A b$ initio calculations}

All magnetic coupling constants have been evaluated by mean of Difference Dedicated Configuration Interaction (DDCI) calculations, ${ }^{68 \mid 69}$ as implemented in the CASDI suite of programs. ${ }^{70171}$ The DDCI method is a multireference approach in which the wavefunction is defined by a combination of Slater determinants built from single and double excitations of the CASSCF wavefuntion. Contrary to the CASSCF approach only the wavefunction coefficients are optimised variationally in the DDCI method, making the calculation dependent on the initial set of orbitals. The excitations are organized according to the number of particles (p) and holes (h) they create in either the inactive or the virtual space, which gives rise to four different levels of calculation: (i) CASCI, which is the reference calculation with no excitations included, (ii) $\mathrm{CAS}+\mathrm{S}$, where only the $1 \mathrm{~h}, 1 \mathrm{p}$ and $1 \mathrm{~h} 1 \mathrm{p}$ excitations are included, (iii) DDC2, where the $2 \mathrm{~h}$ and $2 \mathrm{p}$ excitations are added, and (iv) DDCI, where the $2 \mathrm{~h} 1 \mathrm{p}$ and 1h2p excitations are also included. ${ }^{[55}$ Since the same set of MOs is used to described the different spin states of the system, the $2 \mathrm{~h} 2 \mathrm{p}$ excitations are not included because they do not change the relative energies of the states that are considered. 68169

In the end, the DDCI method is a very accurate variational approach for dynamical correlation, which has already been shown to be effective in quantitatively computing magnetic coupling constants in transition metal compounds of various dimensionalities but also in organic crystals.

In this work, the common set of MOs is generated at the CASSCF level of theory. The MOs are then classified as active, occupied inactive and virtual orbitals, following their occupation in the reference CASSCF wavefunction. To decrease the computational cost, the core orbitals are not included in the DDCI calculations. The size of the active space (CAS $(n, m)$ including $n$ electrons in $m$ orbitals) and the nature of the active orbitals are discussed more deeply in the Results and Discussion section. The CASSCF calculations and 
the integral transformation from atomic to molecular basis sets have been performed with MOLCAS 7.6 code. 72

Additionally, the mechanism of the magnetic coupling has been analyzed on the basis of EXSCI calculations, using the code developed by Maynau and coworkers. ${ }^{73}$ The EXSCI method consists on the rational selection of the determinants employed to build the CI matrix using the exchange integral $\mathrm{K}_{i j}$ as a measure of the interaction between the orbitals involved in the excitations. The procedure requires the use of localized orbitals to make possible a safe truncation. Additionally, also the molecular integrals are truncated, retaining only those integrals larger than a certain threshold. The thresholds used in this work $(s l i=$ 0.0003, sl1 $=0.001$, sl $2=0.0003$ in EXSCI and $s l i 1=0.001$, sli $2=0.0003$ for the molecular integrals) have been previously tested in other systems and are small enough to ensure that the relevant excitations are taken into account.

\section{Structural models}

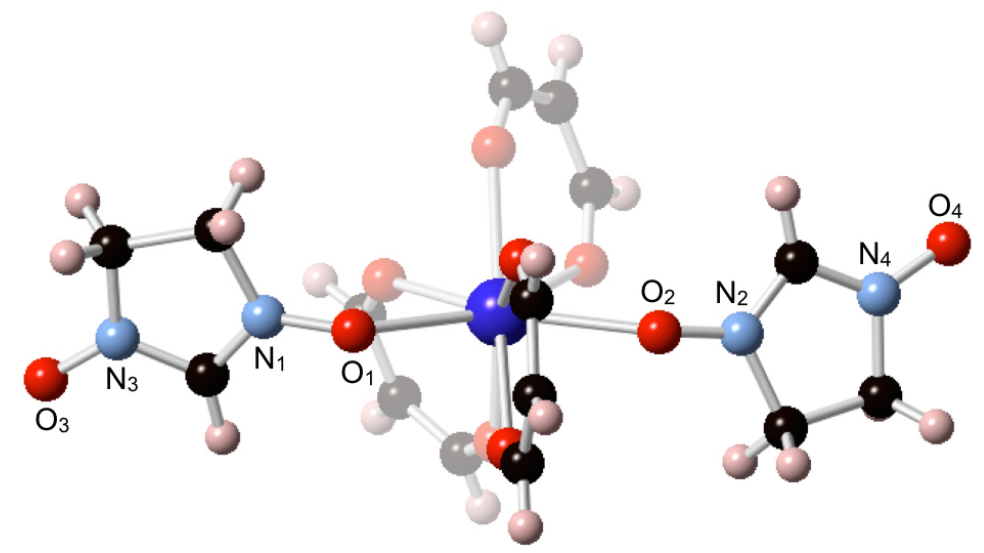

Figure 1: NIT-Y-NIT model for the through-bond NIT-NIT interaction in 1. Black, light blue, red, light pink and dark blue spheres correspond to C, N, O, H and Y atoms, respectively.

For all complexes studied in this work, ab initio calculations were carried out on models built from the X-Ray structures of complexes 1, 2, 3a and 3b, without any further structural optimization. The model employed to study the through-bond NIT-NIT interactions, referred 
hereafter as the NIT-Y-NIT model, is depicted in Figure 1. In this model, the $\mathrm{CF}_{3}$ groups of the $\mathrm{hfac}^{-}$ligands are replaced by hydrogen atoms, as well as the $-\mathrm{R}$ group and the four methyl groups of each NIT radical ligand (respectively in positions -2-, -4- and -5- of the imidazole backbone). A fixed $\mathrm{C}-\mathrm{H}$ distance of $0.93 \AA$ (as in the $\mathrm{hfac}^{-}$ligand) is imposed to all created $\mathrm{C}-\mathrm{H}$ bonds. These structural changes do not to lead to any significant change in the computed properties (see supporting information) whereas they significantly reduce the computational cost and speed-up the calculations.

For the through-space NIT..NIT interactions, the so-called NIT $\cdots$ NIT model only consists of two isolated NIT radical ligands kept in the same relative configuration as in the crystal. As for the NIT-Y-NIT model, the -R group and the four methyl groups of each NIT radical ligand are replaced by $\mathrm{H}$ atoms (except if explicitly notified in the text). We considered as much NIT ...NIT models as potential through-space interaction pathways. For instance, for 1, we considered three possible through-space interaction pathways (Figure 6), with NIT … NIT distances ranging from $3.7 \AA$ to $8.9 \AA$, and thus, three NIT ․ NIT models.

In the calculations, all atoms were described by ANO-RCC basis sets. $\frac{74}{76}$ The following contractions were used: [7s6p4d1f] for the $\mathrm{Y}(\mathrm{III})$ ion, [4s3p1d] for the $\mathrm{N}$ and $\mathrm{O}$ atoms of NIT radical ligands and for the $\mathrm{C}$ atoms in between the two NO groups of each NIT ligand (position -2- of the imidazole backbone), [3s2p] for the remaining $\mathrm{C}$, O and $\mathrm{F}$ (when included) atoms, and $[2 \mathrm{~s}]$ for the $\mathrm{H}$ atoms.

\section{Spin Hamiltonian}

Since the NIT radicals are isotropic half-spin carriers, the exchange interaction between two such ligands can be modeled using the Heisenberg-Dirac-Van Vleck (HDVV) hamiltonian written as follow:

$$
\hat{H}_{H D V V}=-J \hat{S}_{1} \cdot \hat{S}_{2}
$$

where $\hat{S}_{1}$ and $\hat{S}_{2}$ correspond to the spin operators of the two active sites, 1 and 2 , 
and $J$ to the magnetic coupling constant between these sites. In this notation, the coupling constant equals the energy difference between the singlet and triplet states, $J=E(S)-E(T)$. For an antiferromagnetic interaction (i.e. singlet ground state) $J$ is negative while for a ferromagnetic interaction (i.e. triplet ground state), $J$ is positive. Here, one should recall that, intrinsically, exchange interactions are neither ferromagnetic or antiferromagnetic, but correspond to the contribution of several competing ferromagnetic and antiferromagnetic

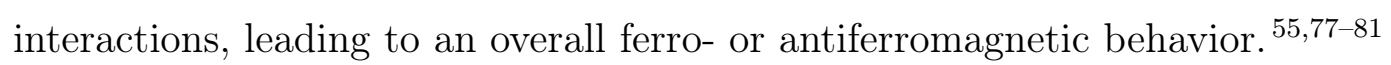

\section{Results and discussion}

\section{Crystal structures and magnetic properties}

Compounds $\mathbf{1}$ and $\mathbf{3 a}$ crystallize in the $\mathrm{P} 21 / \mathrm{n}$ space group, whereas compounds $\mathbf{2}$, and $\mathbf{3 b}$ cristallize in the $\mathrm{P} \overline{1}$ and $\mathrm{P} 22_{1} / \mathrm{c}$ space groups, respectively (Tables $\mathrm{S} 1$ and $\mathrm{S} 2$. For all derivatives, the asymmetric unit is made of one $\operatorname{Ln}(\mathrm{hfac})_{3}$ moiety coordinated to two different NIT radicals that act as monodentate ligands. A NIT-Ln-NIT molecule is then formed. The $\mathrm{Ln}^{3+}$ ion is coordinated by six oxygen atoms of three hfac ${ }^{-}$ligands and two oxygen atoms of the radical ligands to form a fully oxygenated environment $\left(\mathrm{LnO}_{8}\right)$ close to a triangular dodecahedron ( $\mathrm{D}_{2 d}$ site symmetry) (Table $\mathrm{S} 3$. All derivatives show similar Ln-O distances and O-Ln-O angles (Tables $\mathrm{S} 4$ and $\mathrm{S} 5$, and are of the same order of magnitude as in previously reported complexes of this kind. $42|45| 63 \mid 82$

The substitution of the phenyl ring of the NIT radical has a huge effect on the organization of the molecule in the crystal packing (Figure 2). Compounds $\mathbf{2}$ and $\mathbf{3 a}$ present the simplest crystal packing of the series with molecules that are related two-by-two through intermolecular interactions between the uncoordinated NO group of the radicals. However NO distances are quite different with $4.26 \AA$ in $\mathbf{2}$ and $2.80 \AA$ in $\mathbf{3 a}$. The formed supramolecular dimers are thus expected to present different magnetic behaviors. Compounds $\mathbf{1}$ and $\mathbf{3 b}$ present more complex crystal packing as supramolecular chains are created by intermolecular 

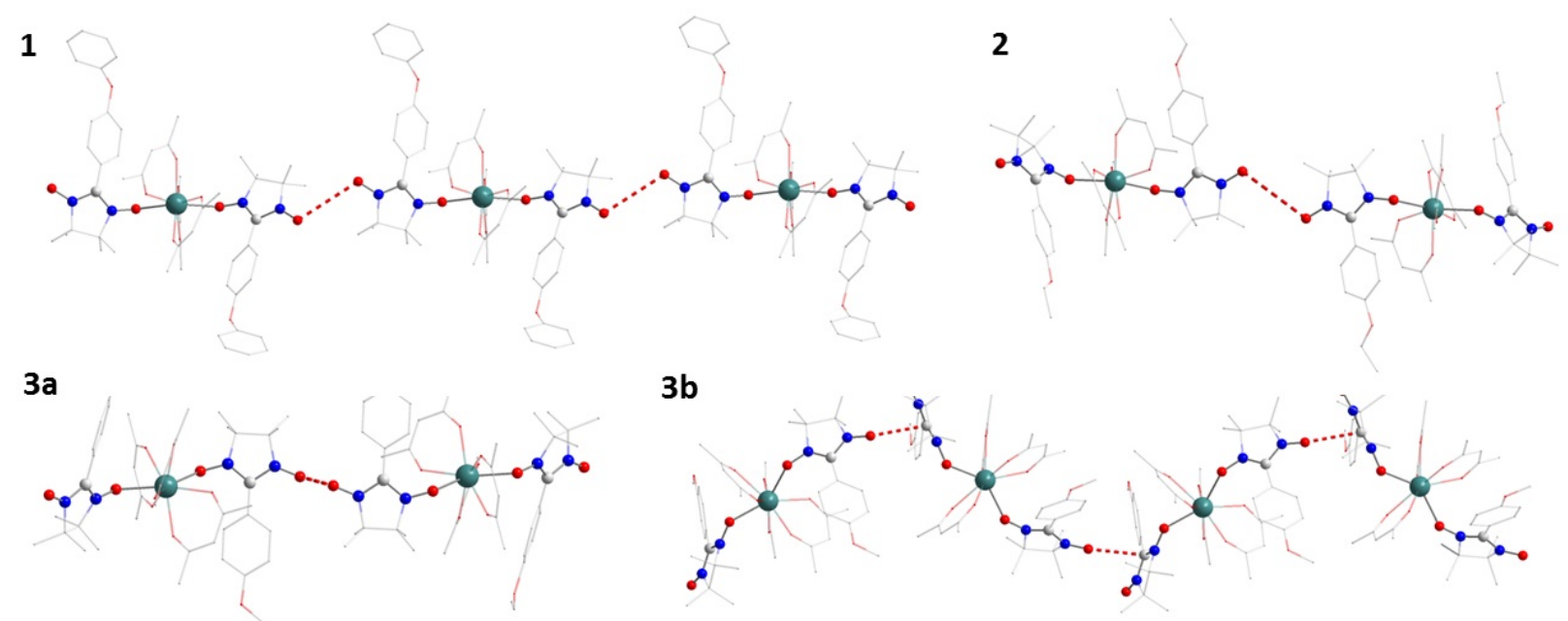

Figure 2: Representation of the $\mathrm{Y}(\mathrm{hfac})_{3}(\mathrm{NIT}-\mathrm{R})_{2}$ derivatives and their supramolecular arrangement ( $\mathbf{1}$ and $\mathbf{3 b}$ : chain-like structures; $\mathbf{2}$ and $\mathbf{3 a}$ tetrameric-like structures). Shortest intermolecular distances $\left(\mathrm{d}_{\text {inter }}\right)$, either $\mathrm{O} \cdots \mathrm{O}$ or $\mathrm{O} \cdots \mathrm{C}$, as dashed red stick. 1: $\mathrm{d}_{\text {inter }}=$ $3.74 \AA ; \mathbf{2}: \mathrm{d}_{\text {inter }}=4.26 \AA ; \mathbf{3 a}: \mathrm{d}_{\text {inter }}=2.80 \AA ; \mathbf{3 b}: \mathrm{d}_{\text {inter }}=2.97 \AA$.

interactions between the NIT-Ln-NIT molecules. These latter entities can be thus considered as "monomers" of either a linear chain in the case of $\mathbf{1}$ or a zig-zag chain in $\mathbf{3 b}$ that have a $\left[\mathrm{Y}(\mathrm{hfac})_{3}(\mathrm{NIT}-\mathrm{R})\right]_{\infty}$ formula.

This latter zig-zag chain shows a very peculiar orthogonal arrangement of the monomers that is possibly responsible of the unexpected reddish color of single crystals of $3 \mathbf{b}$. In fact, each non-coordinating $\mathrm{NO}$ group points at almost $90^{\circ}$ of the imidazole plane of the neighboring radical and is quite close to central carbon of the ON-C-NO moiety (2.97 $\AA$ ). This intermolecular interaction is expected to drastically modify the electronic delocalization within the radical. Finally, intermolecular Ln $\cdots$ Ln distances are $11.34 \AA, 10.07 \AA, 10.54 \AA$, $10.56 \AA$ in $\mathbf{1}, \mathbf{2}, \mathbf{3 a}$ and $\mathbf{3 b}$, respectively.

Magnetic measurements were performed on all complexes (see Experimental section for details). $\chi_{M}$ vs. $\mathrm{T}$ and $\chi_{M} T$ vs. $\mathrm{T}$ experimental curves, with $\chi_{M}$ the molar magnetic susceptibility $\left(\mathrm{cm}^{3} \mathrm{~mol}^{-1}\right)$ and $\mathrm{T}$ the temperature in Kelvin, are shown in Figure 3 . For 1 and $\mathbf{2}$, the experimental susceptometry data are fitted on the basis of the Bleaney-Bowers 

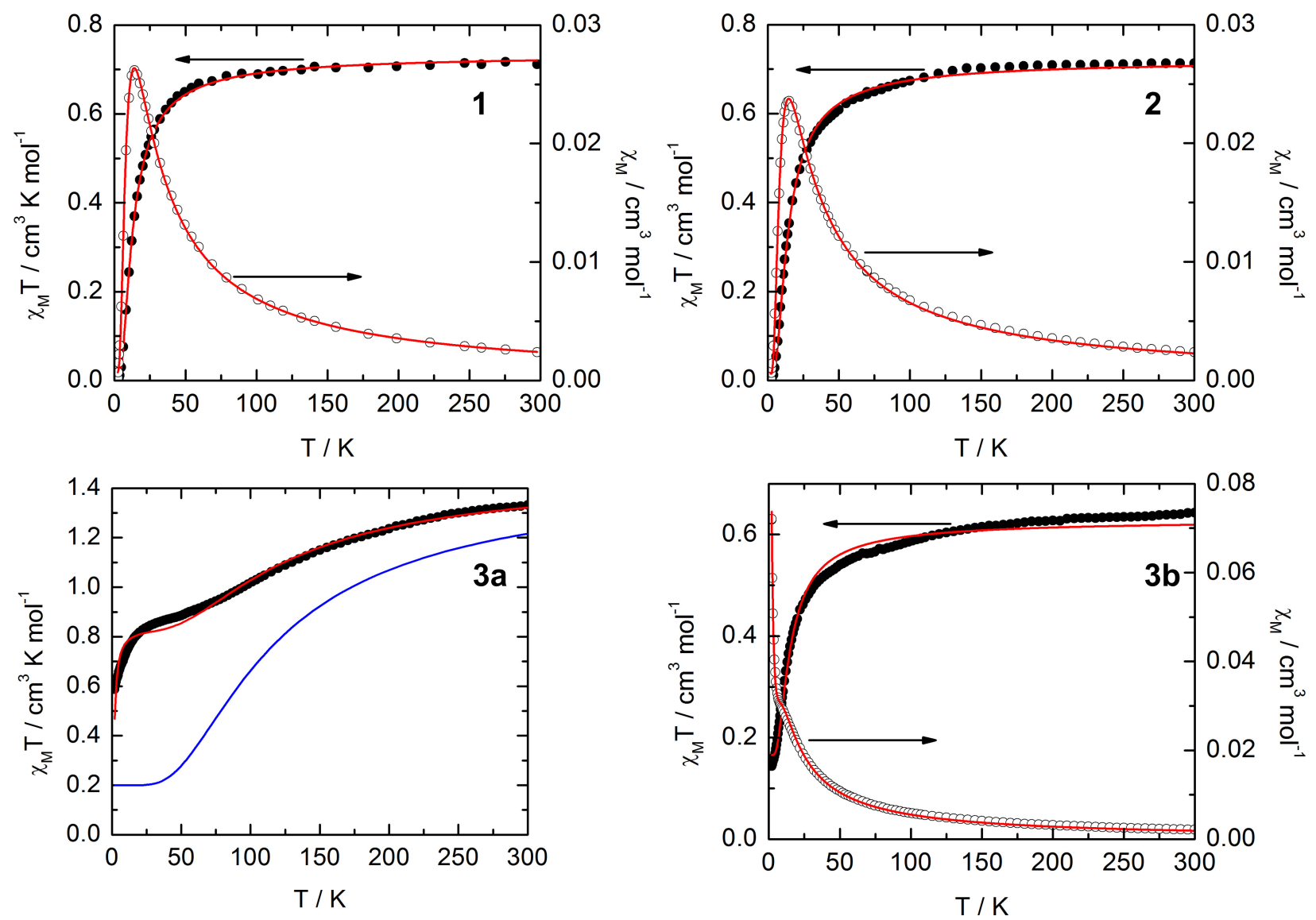

Figure 3: Temperature dependence of $\chi_{M}$ and $\chi_{M} T$ for all complexes. The red line corresponds to the best fitted curve. For $\mathbf{3 a}$, only the temperature dependence of $\chi_{M} T$ is given. The blue line is obtained if $J_{\text {bond }}$ and $J_{\text {space }}$ are inverted in the fitting procedure (see text).

Table 1: Magnetic data obtained from the best fits of $\chi_{M}$ vs. $\mathrm{T}$ and $\chi_{M} T$ vs. $\mathrm{T}$ curves for all complexes.

\begin{tabular}{lcccc}
\hline Compound & $J_{\text {bond }} / \mathrm{cm}^{-1}$ & $J_{\text {space }} / \mathrm{cm}^{-1}$ & $g$ & \% imp $_{\text {im }}$ \\
\hline $\mathbf{1}$ & -15.6 & - & 1.98 & 0.2 \\
$\mathbf{2}$ & -16.5 & - & 1.93 & 0.8 \\
$\mathbf{3 a}$ & -24 & -144 & 1.85 & 15.6 \\
$\mathbf{3 b}$ & -14.9 & - & 1.75 & 25.6 \\
\hline
\end{tabular}


model: 1

$$
\chi_{M}=\left(1-\%_{i m p}\right) \frac{2 N g^{2} \beta^{2}}{k T\left(3+\exp \left(-J_{\text {bond }} / k T\right)\right)}+\%_{i m p} \frac{2 N g^{2} \beta^{2}}{3 k T} s(s+1)
$$

with $J_{\text {bond }}$ the coupling constant between the two spins $\left(-J_{\text {bond }} \hat{s_{1}} \cdot \hat{s_{2}}\right), N$ the Avogadro number, $g$ the Zeeman factor, $\beta$ the Bohr's magneton, $k$ the Boltzmann constant, $T$ the temperature and, finally $\%_{i m p}$, the percentage of paramagnetic impurities with $s=1 / 2$. The best fits (Figure 3) are obtained with values reported in Table 1 with an excellent agreement between experiments and the theoretical model. It shows that no through-space interaction between dimers are required to account for the observed behavior and that sizable interaction is mediated through $\mathrm{Y}(\mathrm{hfac})_{3}$ moiety. For both compounds, the singlet-triplet gap is found about $16 \mathrm{~cm}^{-1} \stackrel{422}{ }$ For $\mathbf{3 b}$, the same fitting procedure leads to poorer agreement with experiment since the sample appears to be contaminated by a relatively large amount of paramagnetic impurities (Figure 3, Table 1). Nevertheless, similar through-bond interaction is found (Table 1) between NIT-PhOMe radicals. It is important to mention at this stage that the Bleaney-Bowers model would be strictly identical if only through-space interactions were considered between radicals. However, since crystal packings of 1, $\mathbf{2}$ and $\mathbf{3 b}$ are different, the fact we extract nearly identical coupling constants certifies that the main interaction pathway is indeed the through-bond one.

Magnetic behavior of $\mathbf{3} \mathbf{a}$ is erratic since on cooling from room temperature $\chi_{M} T$ reaches a plateau below $50 \mathrm{~K}$ before collapsing on further cooling (Figure 3). To explain such a behavior it is absolutely necessary to include more than one interaction term in the model. In other words, intermolecular interactions must be taken into account. Looking at the crystal structure it is reasonable to suppose that relatively large interactions propagate through the short intermolecular distance between two NIT $\left(\mathrm{d}_{\text {inter }}=2.8 \AA\right.$, Figure 2). In this frame the Hamiltonian that can be used is:

$$
\hat{H}=-J_{\text {bond }} \hat{S}_{1} \cdot \hat{S}_{2}-J_{\text {space }} \hat{S}_{2} \cdot \hat{S}_{3}-J_{\text {bond }} \hat{S}_{3} \cdot \hat{S}_{4}
$$


with $J_{\text {space }}$ the through-space coupling constant. We are then considering a linear tetramer of coupled spins. The magnetic susceptibility can be calculated and then compared to experiment by numerical diagonalization of the Hamiltonian matrix. The best fit is represented on Figure 3 with parameters given in Table 1 . Interestingly, $J_{\text {space }}$ is still antiferromagnetic but one order of magnitude larger than $J_{\text {bond }}$, whereas the latter remains of the same order of magnitude than in the three previously discussed compounds. This strengthens our belief in the numerical approach we used. Furthermore, if one suppose that the role of the interactions should be inverted (the stronger within a dimer and the weaker between dimers), the blue curve on Figure 3 is obtained, that is far away from the experimental one. As for $\mathbf{3 b}$, a relatively large amount of paramagnetic impurities must be included to properly reproduce the experiment (Table 1). Since crystals of $\mathbf{3 a}$ and $\mathbf{3} \mathbf{b}$ have been collected by hands, we cannot discard the possibility of pollution of one sample by the other, and thus the larger the amount of $\mathbf{3} \mathbf{a}$ in $\mathbf{3} \mathbf{b}$ (and vice et versa), the smaller the $g$ value.

\section{$A b$ initio determination of the coupling constants for the NIT-NIT interactions in compound 1}

\section{Through-bond NIT-NIT interaction}

An isolated NIT radical possesses three electrons in its valence shell, two of them occupying the antibonding $\pi$ orbitals of the NO groups, and one the $2 \mathrm{p}_{z}$ orbital of the conjugated central $\mathrm{C}$ atom (Scheme 2). The combination of these local orbitals gives rise to three molecular orbitals (MOs), $\phi_{b}, \phi_{S O M O}$ and $\phi_{b}^{*}$, as represented in the right-hand side of Scheme 2. The distribution of the three valence electrons in these MOs gives a doublet ground state. As

discussed elsewhere, $\frac{62183184}{6}$ the largest contribution to the doublet wavefunction corresponds to the determinant with two electrons in the $\phi_{b}$ orbital and one unpaired electron occupying the $\phi_{S O M O}$ orbital.

Hence, the study of the interaction between two NIT radicals can be attempted at first 


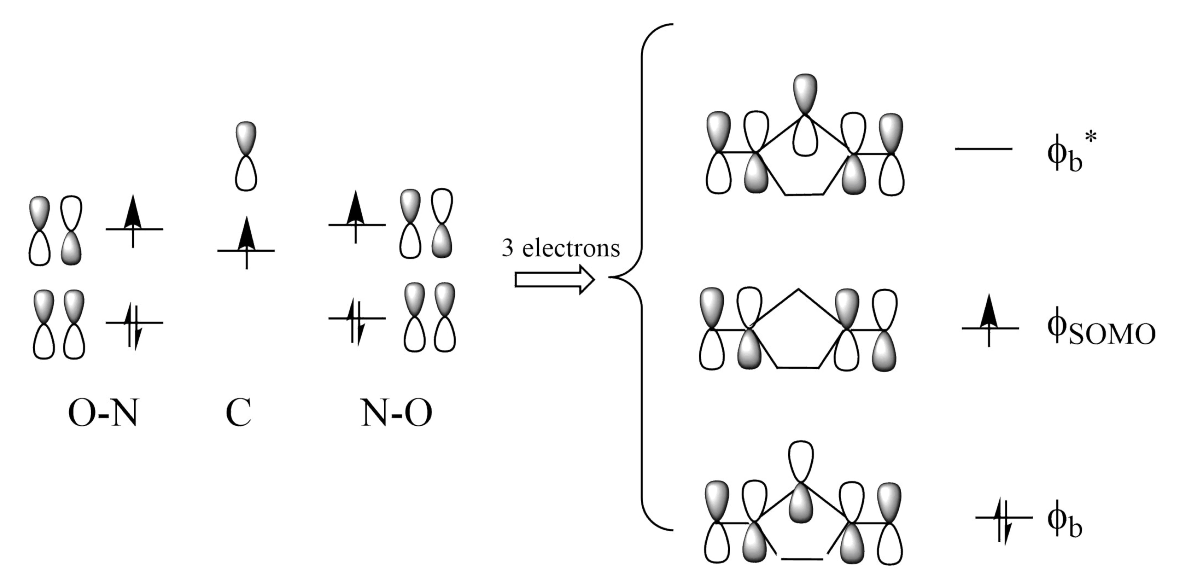

Scheme 2: Schematic representation of the $\pi$ orbitals for an isolated NIT unit. ${ }^{83}$

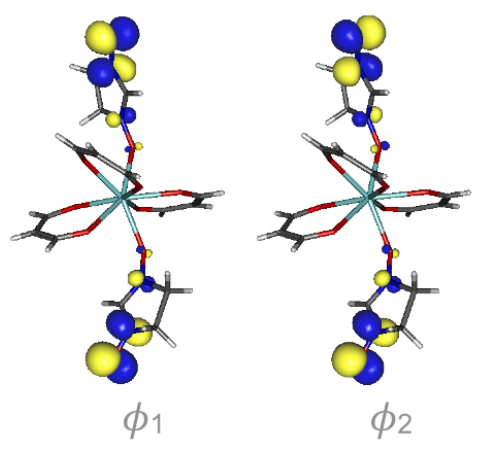

Figure 4: $\mathrm{CAS}(2 / 2) \mathrm{SCF}$ MOs of the excited triplet state for the NIT-Y-NIT model in compound 1. 
using the minimal active space, with two unpaired electrons in the two $\phi_{\text {SOMO }}$ orbitals, i.e. a $\operatorname{CAS}(2 / 2)$. The active MOs are obtained from a $\mathrm{CAS}(2 / 2) \mathrm{SCF}$ calculation on either the ground singlet state $(\mathrm{S})$ or on the excited triplet state $(\mathrm{T})$.

As shown in Figures 4 and $\mathrm{S} 3$, the active MOs correspond to the bonding $\left(\phi_{1}\right)$ and antibonding $\left(\phi_{2}\right)$ combinations of the $\phi_{S O M O}$ orbitals of each NIT group. They are almost identical for the $(\mathrm{S})$ and $(\mathrm{T})$ states, since in both cases, the electronic density is mostly localized on the external NO groups.

The $J_{\text {bond }}$ values computed for the through-bond interaction with these two sets of orbitals are shown in Table 2. Even at the best level of calculation, the ab initio $J_{b o n d}$ values are significantly smaller than that obtained from the fitting procedure. Such strong underestimation is particularly surprising at the DDCI level, where the key effects of dynamical correlation are taken into account and thus leading to $J$ values in good agreement with the experimental values, at least in transition metal complexes. ${ }^{55}$ However, this underestimation correlates well with the strong localization of the electron density on the external NO groups. Previous calculations on organic biradicals presented also this unusual behavior,$\frac{62 \mid 83185186}{1}$ and two different strategies have been adopted in the literature to overcome this problem: (i) to extend the active space by including all the valence $\pi$ electrons and orbitals for each NIT radical, i.e. to use a $\operatorname{CAS}(6 / 6), \underline{62[83 \mid 84}$ and (ii) to still rely on the minimal active space, but using an optimized set of MOs instead of the CASSCF ones which are excessively localized on the external NO moieties. $\frac{85[87][89}{109}$ Here one should mention that increasing the quality of the basis sets, i.e. [4s3p1d] contraction for all $\mathrm{C}$ and $\mathrm{O}$ atoms (see Computational details), induces only a variation of $\sim 5 \%$ of the calculated values.

Table 2 shows the results obtained when using the $\operatorname{CAS}(6 / 6)$ extended active space. As previously, the MOs employed in the CI calculations come from CAS(6/6)SCF calculations on either the singlet ground state or the triplet excited state. The six active MOs of the triplet state are shown in Figure 5. Similar shapes are found for the singlet MOs. Comparing with Figure 4, a larger electron delocalization on the central NO groups is observed, which 
Table 2: Calculated magnetic coupling constants $J_{\text {bond }}\left(\mathrm{cm}^{-1}\right)$ for the NIT-Y-NIT model. In parenthesis, the values obtained with a $\mathrm{CAS}(2 / 2)$ using the $\mathrm{CAS}(6 / 6) \mathrm{SCF}$ MOs. DDCI(6/6) values were not obtained due to computational limitations.

\begin{tabular}{llcccc}
\hline CAS & & Singlet MOs & Triplet MOs & SA-7:3 singlet MOs & Natural DDCI MOs \\
\hline$(2 / 2)$ & CASCI & -0.4 & -0.4 & -1.8 & -1.4 \\
& CAS+S & -1.1 & -1.1 & -3.8 & -4.4 \\
& DDC2 & -1.4 & -1.2 & -5.3 & -5.9 \\
& DDCI & -2.6 & -2.4 & -8.8 & -7.9 \\
\hline$(6 / 6)$ & CASCI & $-0.7(-1.3)$ & $-1.0(-1.3)$ & & \\
& CAS+S & $-1.7(-1.8)$ & $-2.1(-2.6)$ & & \\
& DDC2 & $-2.5(-2.4)$ & $-2.3(-3.0)$ & \\
& DDCI & $-(-4.2)$ & $-(-4.0)$ & \\
\hline$J_{e x p}$ & & \multicolumn{5}{c}{-15.6} & \\
\hline
\end{tabular}

can be directly related to the improvement - with respect to the experimental value - of the calculated $J_{\text {bond }}$ values. Nonetheless, the best estimate $\left(i . e .-4.2 \mathrm{~cm}^{-1}\right.$ ) is still far of being quantitative since it only represents $25 \%$ of the experimental value. It is worth mention that this slight improvement is not related directly to the CAS extension itself but to the larger delocalization of the density observed in the two singly occupied MOs $\left(\phi_{3}\right.$ and $\phi_{4}$ in the top of Figure 5), as demonstrated by the $J_{b o n d}$ values in parenthesis in Table 2. It is also supported by the comparison between the spin density associated to the $\mathrm{CAS}(2 / 2)$ and CAS(6/6) triplet calculations (Figure S4). These $J_{\text {bond }}$ values have been obtained on the basis of the CAS(6/6)SCF MOs, but including in the CI active space only the two singly occupied orbitals (i.e. orbitals $\phi_{3}$ and $\phi_{4}$ ). The present results strongly suggest that the failure of the CI calculation is not related to the size of the active space but to the quality of the MOs.

Thus, we decided to focus on optimizing the MOs within the frame of the minimal CAS. To this end, two options have been explored, both providing quite similar active MOs and hence close $J_{\text {bond }}$ values (see Table 2). First, averaged natural orbitals were built from the density matrices of the singlet and triplet states at the $\operatorname{DDCI}(2 / 2)$ level. The resulting MOs are employed in subsequent DDCI calculations until convergence (iterative DDCI or IDDCI 


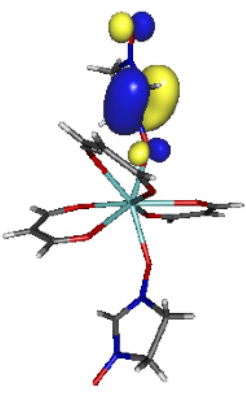

$\phi_{1}$

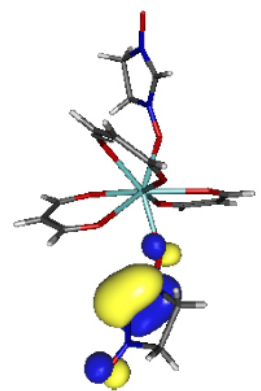

$\phi_{2}$

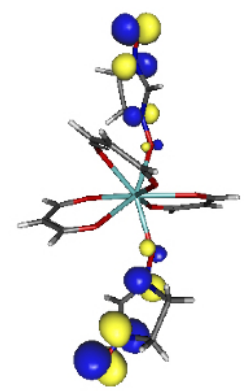

$\phi_{3}$

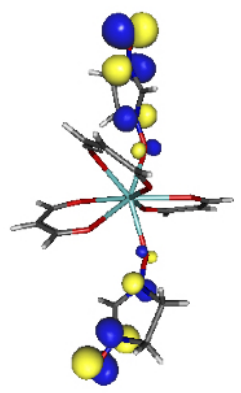

$\phi 4$

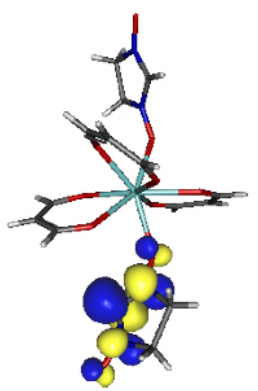

$\phi_{5}$

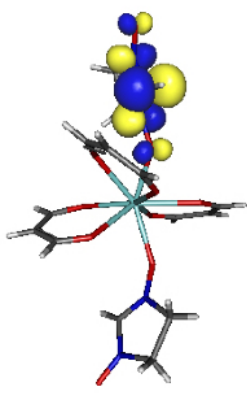

$\phi 6$

(a) $\mathrm{CAS}(6 / 6) \mathrm{SCF}$ singlet MOs

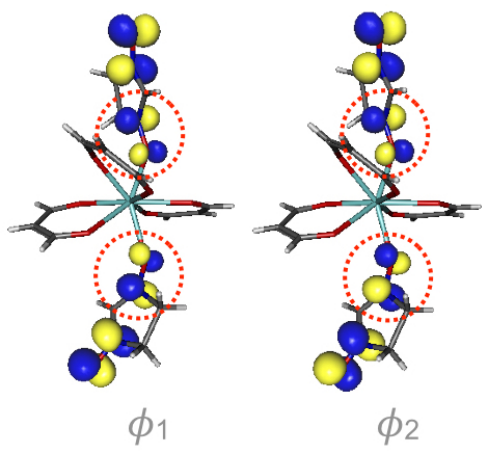

(b) Natural IDDCI(2/2) MOs
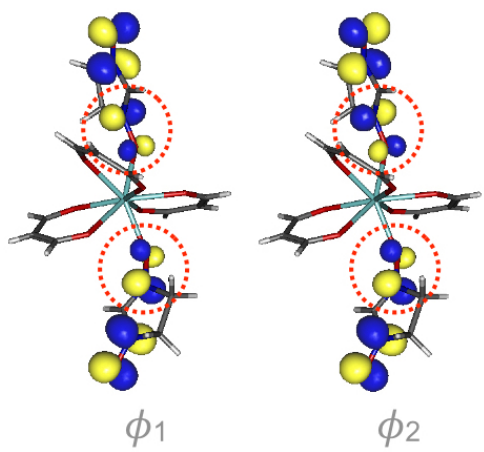

(c) $\mathrm{CAS}(2 / 2) \mathrm{SCF}$ SA 7:3 singlet MOs

Figure 5: (a) CAS(6/6)SCF MOs of the triplet state for the NIT-Y-NIT model in compound 1 with occupations $1.89\left(\phi_{1}\right), 1.88\left(\phi_{2}\right), 1.00\left(\phi_{3}\right), 1.00\left(\phi_{4}\right), 0.11\left(\phi_{5}\right)$ and $0.12\left(\phi_{6}\right) . \phi_{3}$ and $\phi_{4}$ MOs correspond to the symmetric and antisymmetric combinations of the SOMO of each NIT radical. (b) Averaged natural orbitals from the density matrices of the singlet and triplet states at $\mathrm{DDCI}(2 / 2)$ level. (c) $\mathrm{CAS}(2 / 2) \mathrm{SCF}$ MOs of the SA 7:3 singlet wavefunction for the NIT-Y-NIT model in compound 1. 
calculations). ${ }^{90}$ This procedure has been shown to be efficient in eliminating any dependence of the DDCI results on the starting MOs, taking into account the orbital relaxation induced by the electron correlation. The shape of the so-obtained MOs is represented in Figure 5. the most relevant feature being the enhancement of the electron density on the central NO groups, which is in line with the increase of the calculated $J_{\text {bond }}$ value at $\operatorname{DDCI}(2 / 2)$ level (Table 2). Alternatively, Angeli and Calzado have shown recently that it is possible to reproduce the natural DDCI magnetic orbitals with a simple state average CAS $(2 / 2) \mathrm{SCF}$ calculation, that mixes the singlet ground state (essentially represented by the neutral configuration, i.e. with one electron per magnetic site) and the excited ionic singlet states (the two active electrons on the same magnetic site). $\frac{87}{}$ This mixing induces the stabilization of the ionic states, which are usually too high in energy at the CASSCF level, allowing for the relaxation of the active orbitals, and thus providing a nice improvement of the calculated $J$

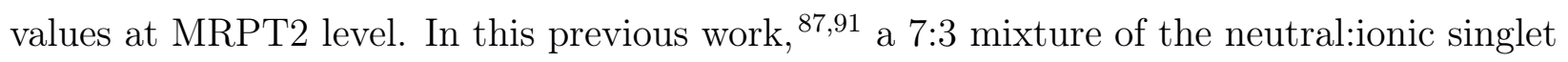
states gave magnetic orbitals showing a large overlap with the DDCI natural MOs. The same mixture was used here and the so-obtained active orbitals are shown in Figure 5, with very similar features to those obtained from the IDDCI approach. The DDCI $J_{b o n d}$ value with these SA-7:3 MOs (i.e. $-8.8 \mathrm{~cm}^{-1}$ ) is then closely similar to that obtained with the natural MOs (i.e. $\left.-7.9 \mathrm{~cm}^{-1}\right)$.

Unfortunately, there are no experimental data available with respect to the spin density in compound 1 or any other of the compounds investigated in the present work, which could validate the shape of the optimized magnetic MOs. Tentative polarized neutron diffraction (PND) measurements were carried out with no concluding results to date. However, for the free NIT radical and for $\mathrm{Cu}(\mathrm{hfac})_{2}(\mathrm{NIT}-\mathrm{Ph})_{2}$ compound, which can be seen as a $\mathrm{Cu}(\mathrm{II})$ analogue of compound 1, such data are available. ${ }^{60 \mid 61}$ In the former case, the spin density is equally delocalized on the two -NO groups of each NIT radical, while in the latter case, it is more delocalized on the outer -NO group of each NIT radical, with only a small contribution on the nitrogen atom of the -NO moiety linked to the $\mathrm{Cu}(\mathrm{II})$ ion. Since the $\mathrm{Cu}(\mathrm{II})$ 
ion interacts rather strongly, i.e. covalently, with the NIT radical ${ }^{61}$ while the Y(III)-NIT interaction is mainly electrostatic, one may expect that the spin density distribution in $\mathbf{1}$ is somewhere in between the two extreme pictures from the free NIT radical on one side and the $\mathrm{Cu}(\text { hfac })_{2}(\mathrm{NIT}-\mathrm{Ph})_{2}$ compound on the other side. Thus, one may be confident in the distribution obtained either with natural or SA MOs (Figure $5 \mathrm{~b}, \mathrm{c}$ ).

In the end, compared to the fitted $J$ value $\left(-15.6 \mathrm{~cm}^{-1}\right)$, our best ab initio estimate $\left(-8.8 \mathrm{~cm}^{-1}\right)$ represents only $55 \%$ of this value, a notably larger deviation than expected for this method, which as already stated, cannot be attributed to the structural simplifications associated to the NIT-Y-NIT model (see Supporting Information).

\section{Through-space NIT. . NIT interactions}

The unexpected disagreement between the calculated and the fitted $J$ values for $\mathbf{1}$ impels us to look for additional exchange interaction pathways able to explain the experimental thermal dependence of the susceptibility curve (Figure 3). Looking at the X-ray structure, three different through-space pathways are explored. They are illustrated in Figure 6. All of them involve two NIT radical groups coordinated to different but close $\mathrm{Y}(\mathrm{hfac})_{3}$ moieties. In these three pathways, the NIT radical ligands are separated by $3.74 \AA, 8.56 \AA$ and 8.90 $\AA$, respectively.

In the particular case of Pathway \#2, to rule out any important contribution of the phenyl rings directly bound to the imidazole backbones to the exchange interaction through $\pi$-stacking interactions or electronic delocalization, two NIT-NIT models are considered: (i) the one classically used in this study (as described in Computational details) and (ii) a second one including explicitly these phenyl rings (one for each NIT radical ligand). The through-space NIT …NIT interactions are evaluated using the approach that was most satisfactorily in the previous section, i.e. $\mathrm{DDCI}(2 / 2)$ on the basis of CASSCF SA-7:3 singlet MOs. For all pathways, the active orbitals correspond to the symmetric and antisymmetric combinations of the SOMOs of each NIT radical (Figure \$5). As for the through-bond 


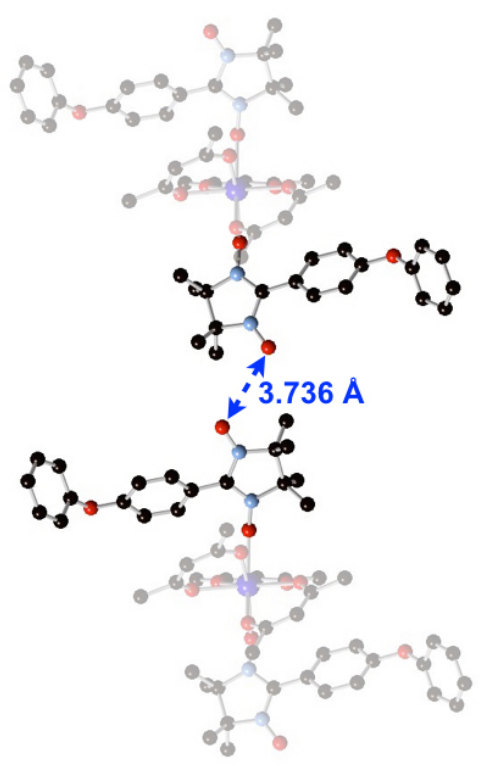

(a) Pathway \#1

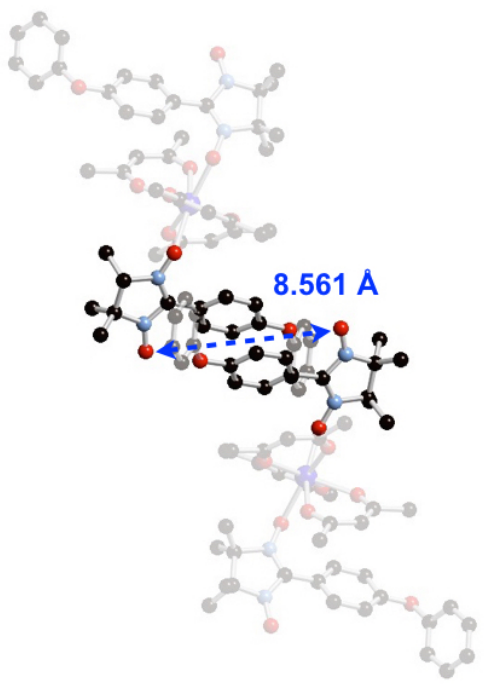

(b) Pathway \#2

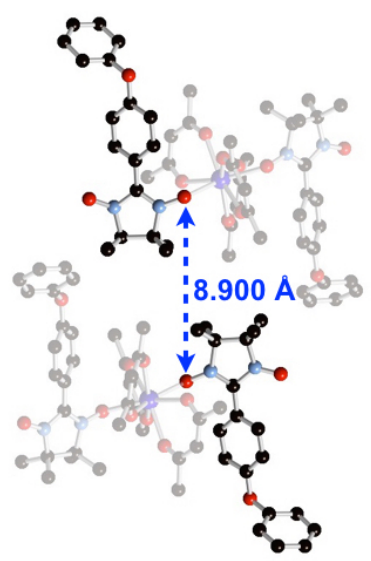

(c) Pathway \#3

Figure 6: Through-space exchange pathways for 1. Same color code as in Figure 1.

NIT-NIT interaction, using the CASSCF SA-7:3 singlet MOs allows for a better description of the magnetic orbitals, with almost equal electron delocalization over the two -NO groups of each NIT radical. For Pathways \#2 (with and without the phenyl rings) and \#3 the computed coupling constants are almost null $\left(-0.0 \mathrm{~cm}^{-1}\right.$ and $+0.1 \mathrm{~cm}^{-1}$, respectively), while for Pathway \#1 the computed coupling constant results in a small but non-negligible antiferromagnetic interaction, with a $J$ value of $-3.6 \mathrm{~cm}^{-1}$. First of all, these results prove the effectiveness of the through-space interactions pathways in the overall magnetic behavior of $\mathbf{1}$ since the intensity of the through-space NIT $\cdots$ NIT interaction is more than $2 / 5$ that of the through-bond NIT-NIT interaction. Though, the strength of the through-space NIT $\cdots$ NIT interaction seems to decrease quite fast with increasing distance, since going from $-3.6 \mathrm{~cm}^{-1}$ to zero while the NIT $\cdots$ NIT distance goes from about $3.5 \AA$ to about $8.5 \AA$.

\section{Fitting of the susceptibility curves}

The presence of a magnetic pathway (Pathway \#1) connecting two neighbor NIT-Y(hfac) $3_{3^{-}}$ NIT units questions the suitability of the Bleaney-Bowers model to fully represent the mag- 
netic interactions in 1, despite the high quality of the fitting based on this model (Figure 3). Indeed, in this model the magnetic behavior can only be attributed to isolated NIT-Y (hfac) ${ }_{3}{ }^{-}$ NIT units where the two NIT radicals are coupled antiferromagnetically, i.e. taking only into account the through-bond NIT-NIT interaction with $J=-15.6 \mathrm{~cm}^{-1}$. On the other hand, the Hatfield model corresponds to a 1D magnetic chain with alternating exchange interactions decribed by two parameters $J_{1}$ and $J_{2}$, such as $J_{2}=\alpha J_{1}$ (see Supporting Information). 1 The green curve in Figure 7 is obtained with this model using the calculated through-bond and through-space $J$ values, i.e. $J_{\text {bond }}=-8.8 \mathrm{~cm}^{-1}$ and $J_{\text {space }}=-3.6 \mathrm{~cm}^{-1}$, respectively, and $\alpha$ $=0.409$. The fact that this curve predicts a larger $\chi$ value than the experimental one indicates that the magnetic interactions are underestimated in our computational protocol. By increasing these values by a factor of 1.5 (i.e. with $J_{\text {bond }}$ and $J_{\text {space }}$ raised to $-13.2 \mathrm{~cm}^{-1}$ and $-5.4 \mathrm{~cm}^{-1}$, respectively) a better agreement with the experimental $\chi$ vs $\mathrm{T}$ curve is obtained (blue curve in Figure 7). This result suggests that, even at the DDCI level of calculation, the computed $J$ values are underestimated by about $35 \%$. 


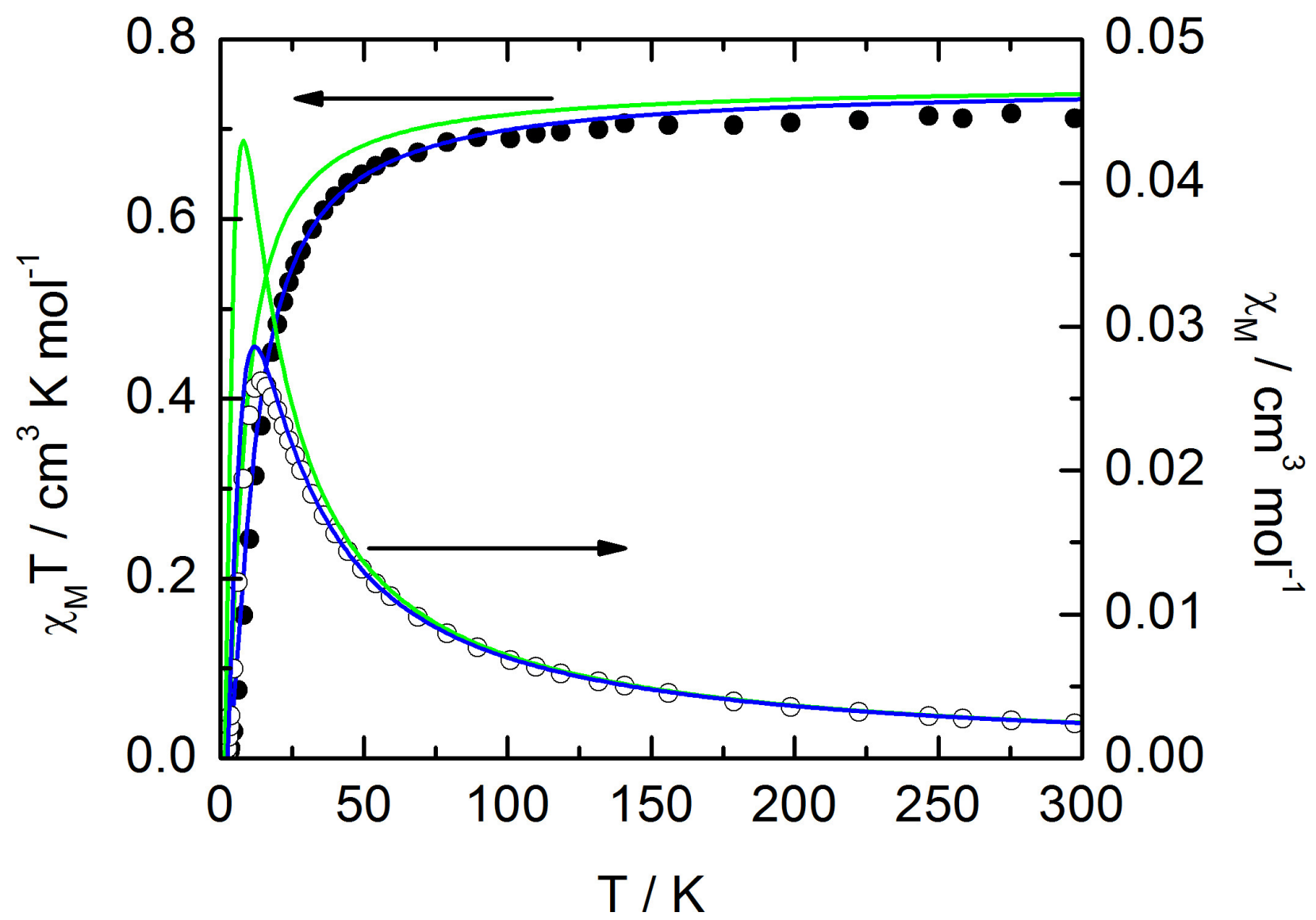

Figure 7: Plots of $\chi_{M}$ and $\chi_{M} T$ as a function of temperature for compound 1 with simulated curves using the Hatfield model and the calculated $J$ values (green curve; $J_{b o n d}=-8.8 \mathrm{~cm}^{-1}$, $J_{\text {space }}=-3.6 \mathrm{~cm}^{-1}$ ) and $J * 1.5$ values (blue curve; $J_{\text {bond }}=-13.2 \mathrm{~cm}^{-1}$ and $J_{\text {space }}=-5.4$ $\left.\mathrm{cm}^{-1}\right)$. Both curves have been simulated using the experimental $g$ value $(g=1.98)$.

To summarize, this result indicates that (i) for both the through-bond and through-space NIT-NIT interactions, the computed $J$ values are underestimated; (ii) once the calculated $J$ values are corrected, a model based on an alternating 1D chain can accurately reproduce the experimental dependence of the magnetic response with temperature; (iii) the BleaneyBowers model has to be considered as an effective model, able to reproduce the overall experimental behavior, but not as a realistic picture of the different interactions operating in 1. 


\section{The $\mathrm{Y}\left(\mathrm{NO}_{3}\right)_{3}(\mathrm{NIT} \text {-triazol })_{2}$ compound}

The underestimation of the $J$ values in $\mathbf{1}$, while puzzling, is not an isolated case. We have found the same trend for another NIT-based complex, i.e. the $\mathrm{Y}\left(\mathrm{NO}_{3}\right)_{3}(\mathrm{NITtriazol})_{2}$ (Figure

S6) that has been investigated in-depth in the late 90's by Kahn ${ }^{92193}$ and Sutter. ${ }^{9495}$ Contrary to the other compounds investigated in the present work, in this complex, the NIT-derived radicals act as bidentate ligands. Also, the $\mathrm{Y}(\mathrm{III})$ ion is in a $\mathrm{N}_{2} \mathrm{O}_{8}$ environment, with six oxygen atoms coming from three $3 \eta^{2}-\mathrm{NO}_{3}$ ligands while the two remaining oxygen atoms and the two nitrogen atoms come from the two NIT-derived radical ligands. Finally, the large intermolecular NIT …NIT distances (around $6 \AA$ ) suggests the absence of any through-space NIT $\cdots$ NIT interaction. This is supported by the fit of the experimental data, which give an intermolecular interaction of $0.009 \mathrm{~cm}^{-1}$ when taken into account, whereas the experimental coupling constant of the through-bond NIT-NIT interaction is found at $-3.1 \mathrm{~cm}^{-1} . \underline{94}$ Using the same computational settings as previously and a NIT-Y-NIT model where all six methyl groups of each NIT-derived radical ligand are replaced by $\mathrm{H}$ atoms, the $J$ value for the through-bond NIT-NIT interaction is computed (Table S7). Here again, the spin density, and thus the shape of the magnetic MOs (Figure \$7), seem to be a key parameter in the calculated values. At the best level of calculation $(\mathrm{DDCI}(2 / 2)$ on the basis of SA-7:3 singlet MOs), the computed $J_{\text {bond }}$ value $\left(J_{\text {bond }}=-2.09 \mathrm{~cm}^{-1}\right)$ is again underestimated, by roughly $30 \%$ with respect to the experimental value. Till no opposite evidence has been given, one might thus consider this trend to be systematical for NIT-based complexes of this kind.

\section{Mechanisms governing the coupling in $\mathrm{Y}(\mathrm{hfac})_{3}(\mathrm{NIT}-\mathrm{PhOPh})_{2}(1)$}

The mechanisms governing the NIT-NIT magnetic interaction through a rare earth ion, i.e. in the NIT-RE(hfac) $)_{3}$-NIT complexes with $\mathrm{RE}=\mathrm{Gd}(\mathrm{III}), \mathrm{Eu}(\mathrm{III}), \mathrm{Y}(\mathrm{III})$ have been addressed by different authors in the past. $\frac{2512745547966}{4 h r e e}$ main mechanisms have been invoked: (i) the direct interaction between NIT units, (ii) the super-exchange mediated by the $\mathrm{hfac}^{-}$ligands and (iii) the super-exchange mediated by the RE ion. In this latter case, 
depending on the nature of the RE ion, different mechanisms have been suggested involving the highest occupied orbitals (4p for Y, 4f for Gd and Eu), virtual orbitals (4d and 5s for Y, $5 \mathrm{~d}$ and $6 \mathrm{~s}$ for $\mathrm{Eu}$ and $\mathrm{Gd}$ ) or the partially occupied $4 \mathrm{f}$ orbitals for $\mathrm{Gd}$ and $\mathrm{Eu}$ ions.

In the particular case of the Y(III) complexes we are interested in, mechanism (i) can be eliminated due to the rather large distance between the NIT radicals (around $6 \AA$ ). For mechanism (ii), the p orbitals of the oxygen atoms belonging to the $\mathrm{hfac}^{-}$ligand perpendicular to the $\mathrm{O}_{1}-\mathrm{O}_{2}$ direction, later referred as central hfac ${ }^{-}$ligand (Figure 1), are assumed to be 90 degrees away from the magnetic MOs, which ensures significant orbital overlap, and thus a strong antiferromagnetic contribution (according to Goodenough and Kanamori's rules). ${ }^{49}$ The same goes if one considers that the $4 \mathrm{p}$ orbitals of the Y(III) ion are involved. Finally, for mechanism (iii), super-exchange is expected to give an antiferromagnetic contribution through spin-polarisation in the empty $4 \mathrm{~d}$ and/or 5s orbitals. In their inspiring work, Benelli and Gatteschi had a preference for the mechanisms involving either the occupied or the virtual orbitals of the Y(III) ion. ${ }^{25 / 45}$ However, a definitive demonstration is still lacking.

With this in mind, we have used different strategies to elucidate the mechanism(s) actually operating in $\mathbf{1}$, by isolating each of the effective mechanisms and estimating their contribution to the whole through-bond NIT-NIT interaction. In all these calculations, a minimal active space, i.e. $\mathrm{DDCI}(2 / 2)$, is employed based on the CASSCF SA-7:3 singlet MOs.

The contribution of direct exchange can be roughly evaluated using a simplified $N I T-Y_{-}$ NIT model where all the atoms of the three $\mathrm{hfac}^{-}$groups and the Y(III) ion are eliminated, i.e. only the two NIT radicals are considered, at the same distance and relative orientation as in the crystal structure. The $J_{\text {bond }}$ value obtained at DDCI $(2 / 2)$ level is of $-0.1 \mathrm{~cm}^{-1}$. This result confirms the hypothesis of Benelli et al., ${ }^{\sqrt[25 \mid 45]{14}}$ which ruled out this direct exchange mechanism due to the large intra-molecular NIT . . NIT distances in these systems.

To analyze the role of the Y(III) ion and hfac $^{-}$ligands in the super-exchange mechanisms, the whole set of CASSCF SA-7:3 MOs has been localized ${ }^{97}$ and a new series of calculations 
Table 3: Calculated magnetic coupling constants $J_{\text {bond }}\left(\mathrm{cm}^{-1}\right)$ for the NIT-Y-NIT model of complex 1 using localized orbitals and various orbital selections through the EXSCI approach. Reference value (without truncation) is $J_{\text {bond }}=-8.9 \mathrm{~cm}^{-1}$.

\begin{tabular}{lc}
\hline Selection & $J_{\text {bond }}$ \\
\hline whole set of orbitals & -7.2 \\
without Y(III) orbitals & -6.4 \\
without all hfac ${ }^{-}$orbitals & -4.9 \\
without central hfac ${ }^{-}$orbitals & -5.2 \\
without all hfac & - Y Y (II) \\
and occ. and virt. NIT $\pi$ orbitals & -2.7 \\
\hline
\end{tabular}

has been performed eliminating step-by-step the orbitals localized on these groups. The procedure benefits from the EXSCI approach, that considerably reduces the computational cost by excluding all the non-essential excitations by means of a threshold on the related exchange interaction integrals. ${ }^{73}$ Details of this method and recent applications can be found in Refs. 73, 98 101. Within the frame of the EXSCI approach, the reference $J_{\text {bond }}$ value is now $-7.2 \mathrm{~cm}^{-1}$ to be compared to $-8.9 \mathrm{~cm}^{-1}$ obtained without truncation (Table 3). If all the orbitals centered on the $\mathrm{Y}(\mathrm{III})$ ion are eliminated, the $J_{b o n d}$ value goes down to $-6.4 \mathrm{~cm}^{-1}$. This small reduction of $J_{b o n d}$ excludes the super-exchange mechanism via the occupied $4 \mathrm{p}$ or virtual $4 \mathrm{~d}$ or 5 s orbitals of Y(III) ion as the main 'pathway' for the magnetic interaction. If instead, the orbitals centered on the three $\mathrm{hfac}^{-}$ligands are eliminated, the $J_{b o n d}$ value goes down to $-4.9 \mathrm{~cm}^{-1}$, while if only the orbitals of the central $\mathrm{hfac}^{-}$ligand are eliminated $J_{\text {bond }}=-5.2 \mathrm{~cm}^{-1}$ (Table 3). This clearly suggests the implication of the hfac ${ }^{-}$ligands in the super-exchange mechanism. In particular, the central hfac ${ }^{-}$actively participates to this mechanism (Scheme 3). Into more details, when looking at the DDCI wavefunction, it appears that the excited configurations with a significant weight can be described as charge transfer forms, related to the neutral ones by single excitations. In fact, as shown in Scheme 3 in the first step, one electron is promoted from one of the $\mathrm{hfac}^{-}$orbitals to the magnetic orbital located on one of the NIT radical. In other words, there is a charge transfer from the ancillary ligand to the NIT radical. Then, one electron from the second NIT radical 
moves to the hfac ${ }^{-}$orbital. This corresponds to the formation of an ionic form, where the two active electrons occupy simultaneously the same magnetic center. Next, one electron moves from the hfac ${ }^{-}$ligand to the NIT radical, changing its spin. Finally, the neutral form is restored (Scheme 3). This mechanism is responsible for an antiferromagnetic contribution to the whole magnetic coupling, such as

$$
J_{A F} \propto-4 \frac{\left(t_{C T}^{N}\right)^{2} \cdot\left(t_{C T}^{I}\right)^{2}}{\Delta E_{C T}^{2} \cdot U}
$$

where $t_{C T}^{N}$ corresponds to the interaction between the charge transfer and the neutral form and $t_{C T}^{I}$ is the interaction between the charge transfer and the ionic form. $\Delta E_{C T}$ and $U$ are the relative energy of the CT and ionic forms, respectively (Scheme 3). $551102[104$

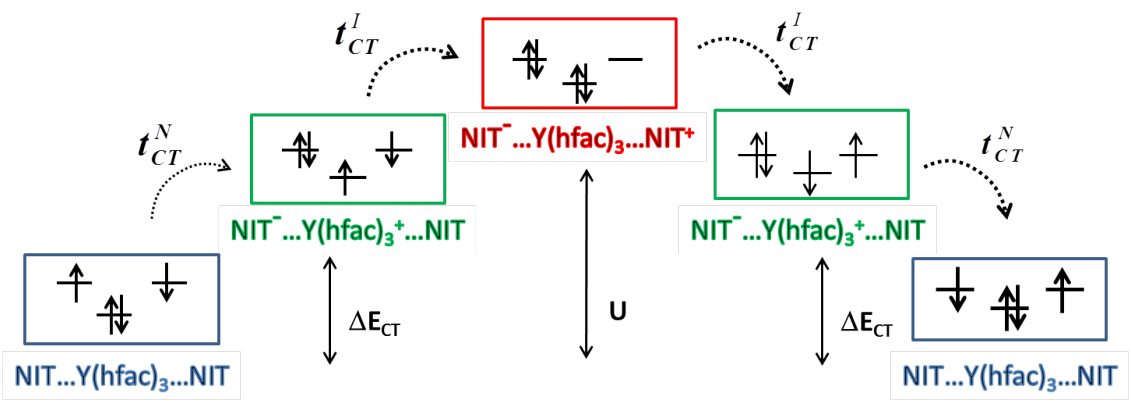

Scheme 3: Super-exchange mechanism involved in the through-bond NIT-NIT magnetic interaction in 1: neutral (blue), ionic (red) and CT (green) forms.

If, in addition to the $\mathrm{hfac}^{-}$and Y(III) orbitals, the occupied and virtual $\pi$ orbitals centered on the NIT radicals (orbitals $\phi_{1}, \phi_{2}, \phi_{5}$ and $\phi_{6}$ in Figure 5) are eliminated, the $J_{\text {bond }}$ value drops now to $-2.7 \mathrm{~cm}^{-1}$ (Table 3). These orbitals centered on the NIT radicals participate in the $1 \mathrm{~h} 1 \mathrm{p}$ excitations that promote one electron from the NIT occupied $\pi$ orbital to the virtual $\pi^{*}$ orbital, which contribute to the relaxation of the ionic and CT forms, reducing their energies $\Delta E_{C T}$ in Eq. 4. This provides an antiferromagnetic contribution to the overall magnetic coupling.

Furthermore, it is possible to isolate the effect of these $1 \mathrm{~h} 1 \mathrm{p}$ excitations by performing a class-partitioned $\mathrm{CI}$ calculation. ${ }^{83104-106}$ In this case, only a set of excitations is included 
in the CI matrix. Its diagonalization gives the variational impact of the selected set of excitations on the coupling constant. The diagonalization of the CAS $+1 \mathrm{~h} 1 \mathrm{p}$ space, that is, with only the CAS determinants (neutral and ionic forms) and the 1h1p excitation, gives a $J_{\text {bond }}$ value of $-5.6 \mathrm{~cm}^{-1}$ to be compared to the value obtained with the whole DDCI space, i.e. $-8.9 \mathrm{~cm}^{-1}$. The CAS $+1 \mathrm{~h} 1 \mathrm{p}$ space represents about $40 \%$ of the whole DDCI space, meaning that the $1 \mathrm{~h} 1 \mathrm{p}$ excitations themselves bring an important contribution to the antiferromagnetic coupling constant, that is enhanced when interacting with the CT determinants in the DDCI space. Among the 1h1p excitations, those involving the $\pi$ occupied and virtual MOs of the NIT radicals are the key ones. In fact, if the two occupied orbitals $\phi_{1}$ and $\phi_{2}$ (Figure 5) are eliminated, the $J_{b o n d}$ value at the CAS $+1 \mathrm{~h} 1 \mathrm{p}$ level is reduced to $-0.7 \mathrm{~cm}^{-1}$ and finally becomes ferromagnetic with a value of $+5.9 \mathrm{~cm}^{-1}$ at the DDCI level.

In summary, the subtle mechanism at the origin of the antiferromagnetic through-bond NIT-NIT interaction can be seen as a two-step mechanism involving both the $\pi$ orbitals of the NIT radicals and the $\pi$ orbitals of the central hfac ${ }^{-}$ligand (the one perpendicular to the $\mathrm{O}_{1}-\mathrm{O}_{2}$ direction, Figure 1). First, there is a charge transfer from the $\mathrm{hfac}^{-}$to the NIT radical that can relax by interacting with the local excitations on the NIT radical (from occupied to virtual MOs). Then, a second charge transfer operates from the other NIT radical to the hfac $^{-}$orbitals. In the end, the operative exchange mechanism is not simply a classical superexchange mechanism through the bridging $\mathrm{hfac}^{-}$ligand, but a more complex combination of this super-exchange with simultaneous stabilizing local excitations on the radical units.

\section{Extension to complexes 2, 3a and $3 \mathrm{~b}$}

\section{Through-bond NIT-NIT interaction}

In order to test the robustness of our ab initio approach, we have extended our study to the closely related complexes $\mathbf{2}, \mathbf{3} \mathbf{a}$ and $\mathbf{3} \mathbf{b}$, i.e. with a very similar coordination environment

but highly different supramolecular arrangements. Evaluation of the magnetic coupling constants for the through-bond NIT-NIT interaction in compounds $\mathbf{2}, \mathbf{3 a}$ and $\mathbf{3} \mathbf{b}$ has been 
carried out using the same procedure as for compound $\mathbf{1}$. The results are collected in Table 4. As expected, the $J_{b o n d}$ values are all of the same order of magnitude. However, they can be separated in two groups: about $-8.5 \mathrm{~cm}^{-1}$ for $\mathbf{1}$ and $\mathbf{3 a}$ and about $-10.2 \mathrm{~cm}^{-1}$ for $\mathbf{2}$ and 3b. From the structural parameters (Tables $\$ 44$ and $\$ 55$, this difference can be explained by the slightly different NIT-NIT angle. For $\mathbf{1}$ and $\mathbf{3 a}$, this angle is about $140-145^{\circ}$ while for $\mathbf{2}$ and $\mathbf{3 b}$ it is close to $135^{\circ}$. The connexion between this angle and the value of $J_{b o n d}$ can be analyzed in terms of orbital overlap: the larger this angle, the smaller the overlap between the orbitals of the NIT radical ligands and the $\mathrm{hfac}^{-}$ligand perpendicular to the $\mathrm{O}_{1}-\mathrm{O}_{2}$ direction (Scheme $\mathrm{S} 1$, and thus, the lower the antiferromagnetic contribution. From a purely theoretical point of view, this suggests that even larger values of $J$ could be obtained by reducing the angle between the NIT radical ligands, a hardly predictable point from the synthetic point of view.

Table 4: Through-bond $\left(J_{\text {bond }}\right)$ and through-space $\left(J_{\text {space }}\right)$ calculated magnetic coupling constants $\left(\mathrm{cm}^{-1}\right)$ for complexes 1, 2, 3a and $\mathbf{3 b}$. Experimental values are given for comparison. All the calculations are performed at the $\operatorname{DDCI}(2 / 2)$ level, using state average singlet 7:3 MOs.

\begin{tabular}{ccccc}
\hline Compound & $J_{\text {bond }}$ & $J_{\text {bond/exp }}$ & $J_{\text {space }}$ & $J_{\text {space/exp }}$ \\
\hline $\mathbf{1}$ & -8.7 & -15.6 & -3.6 & - \\
$\mathbf{2}$ & -10.2 & -16.5 & -0.5 & - \\
$\mathbf{3 a}$ & -8.5 & -24.0 & -178.0 & -144.0 \\
$\mathbf{3 b}$ & -10.2 & -18.5 & 19.6 & - \\
\hline
\end{tabular}

\section{Through-space NIT $\cdots$ NIT interaction}

Regarding the through-space NIT $\cdots$ NIT interactions in $\mathbf{2}, \mathbf{3 a}$ and $\mathbf{3 b}$, only those where the NIT...NIT distance is smaller than $5 \AA$ have been considered. As shown in Figure 8 , only one through-space pathway has been investigated for each compound. Magnetic orbitals are shown in Figure 9.

For $\mathbf{2}$ and $\mathbf{3 a}$, the NIT interacting radicals adopt a head-to-tail conformation, as in $\mathbf{1}$, but this time, they lie in almost parallel planes. The shortest inter-molecular NIT $\cdots$ NIT 


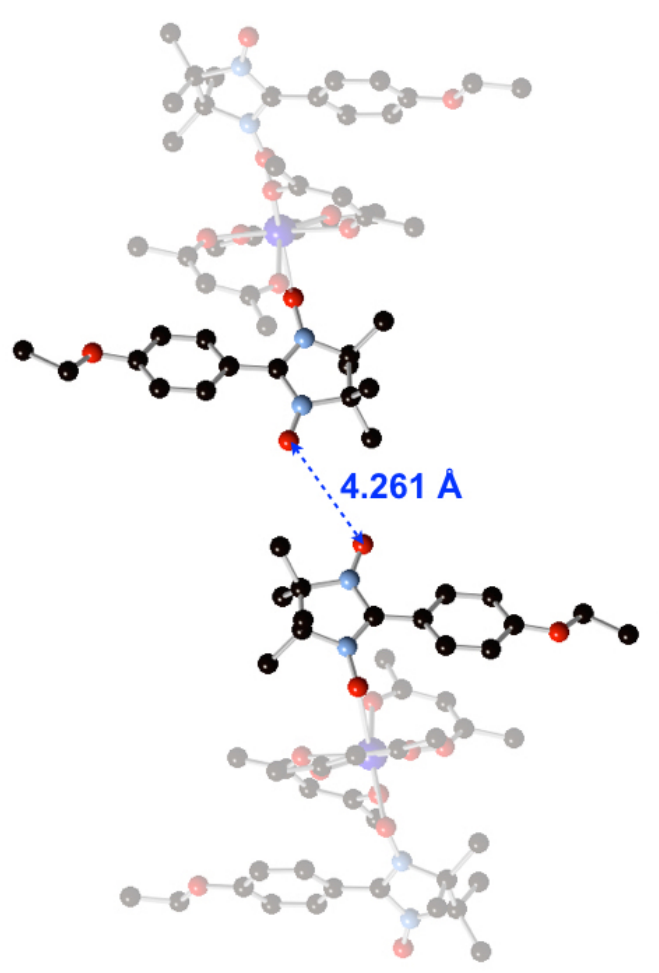

(a)

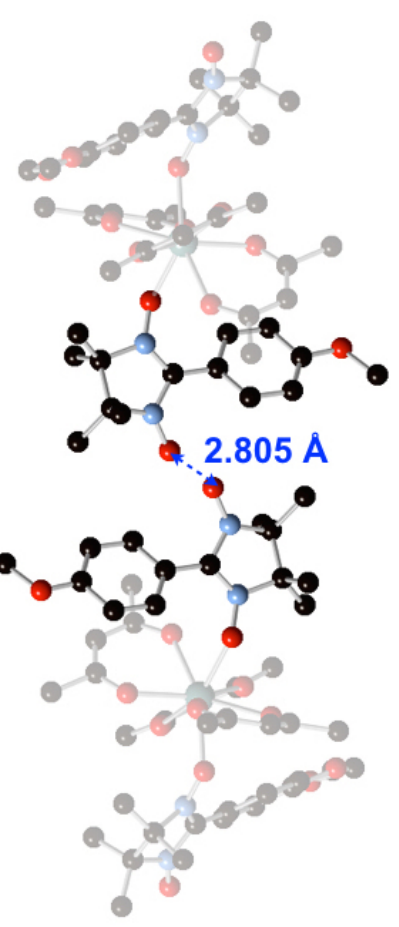

(b)

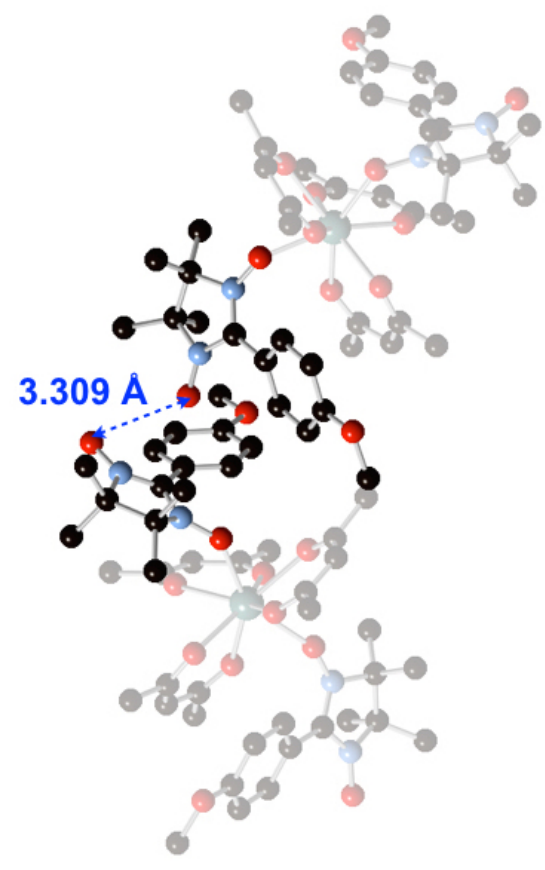

(c)

Figure 8: Through-space pathways (O $\cdots$ O distances in blue) for $\mathbf{2}(\mathrm{a}), \mathbf{3 a}(\mathrm{b})$ and $\mathbf{3 b}(\mathrm{c})$.

distances are $4.261 \AA$ and $2.805 \AA$ for $\mathbf{2}$ and $\mathbf{3 a}$, respectively. For $\mathbf{2}$, the calculated coupling is very weak $\left(J_{\text {space }}=-0.5 \mathrm{~cm}^{-1}\right)$, as expected for such a large separation between the NIT units. From this, the Bleaney-Bowers model used experimentally seems to be appropriate to analyse the magnetic behavior of compound $\mathbf{2}$. However, as for $\mathbf{1}$, the magnetic curves can also be simulated by taking into account all the calculated magnetic interactions using the Hatfield model (Figure S8). Here again, the calculated $J$ values have to be slightly corrected for a better agreement with experiment.

For $3 \mathbf{a}$, on the other hand, the through-space NIT $\cdots$ NIT interaction is far from negligible since a strong antiferromagnetic interaction is found $\left(J_{\text {space }}=-144 \mathrm{~cm}^{-1}\right.$, Table 4 ). This is undoubtedly related to the very short distance and the $180^{\circ}$ between the planes containing the NIT radicals, that favors an efficient overlap between the $\pi$ orbitals (Figure 9). Importantly, these results well support the experimental interpretation with a much larger through-space than through-bond interaction (Table 4). 


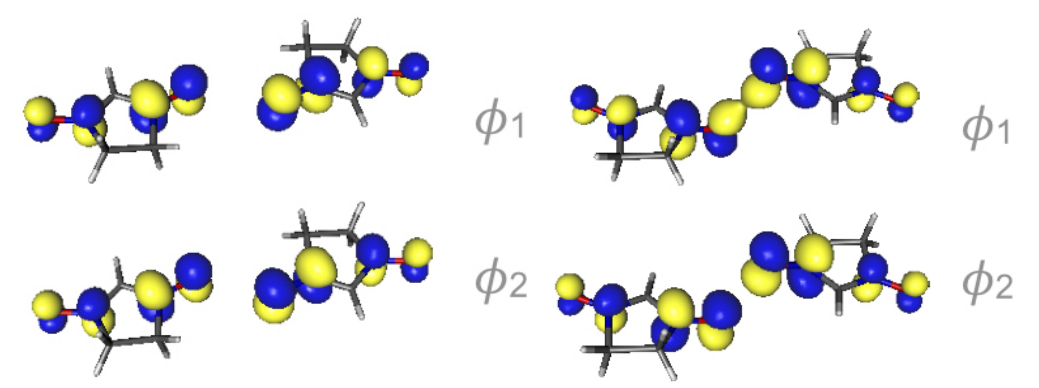

(a)

(b)

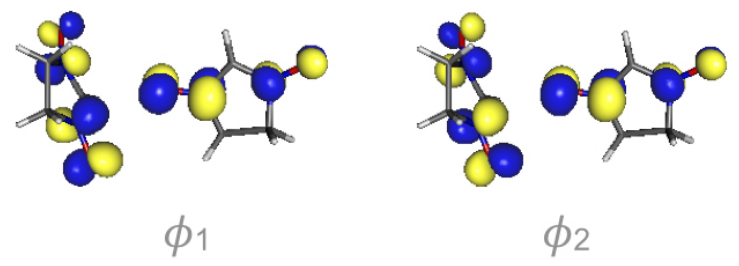

(c)

Figure 9: CAS(2,2)SCF SA-7:3 singlet MOs $\left(\phi_{1}\right.$ and $\left.\phi_{2}\right)$ for the through-space NIT $\cdots$ NIT interaction in $\mathbf{2}(\mathrm{a}), \mathbf{3 a}(\mathrm{b})$ and $\mathbf{3} \mathbf{b}(\mathrm{c})$.

For $\mathbf{3 b}$, as shown in Figure 8, the two interacting NIT radicals lie perpendicularly and the shortest inter-molecular NIT . . NIT distance is $3.309 \AA$. Here, the calculated interaction is found ferromagnetic with a $J_{\text {space }}$ value of $19.6 \mathrm{~cm}^{-1}$ (Table 4 ). This quite large ferromagnetic interaction is induced by the orthogonality of the magnetic orbitals (Figure 9). Remarkably, this through-space NIT... NIT interaction is of the same order of magnitude as the throughbond NIT-NIT interaction $\left(J_{\text {bond }}=-10.2 \mathrm{~cm}^{-1}\right)$. Similarly to $\mathbf{1}$, from the magnetic point of view, $\mathbf{3 b}$ can thus be seen as a magnetic $1 \mathrm{D}$ chain with alternating interactions, but this time, with both ferromagnetic (intermolecular) and antiferromagnetic (intramolecular) interactions. 107 The curve simulated with these calculated $J$ values (Figure $\mathrm{S} 9$ ) does not reproduce the experimental one as good as for $\mathbf{1}$ and $\mathbf{2}$. However, this behavior was certainly expected for two well-separated reasons. From the computational point of view, it is well

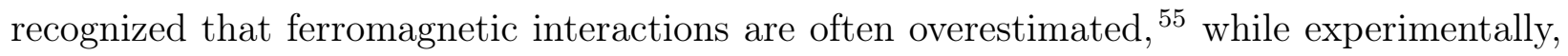
it has been evidenced (See Magnetic properties Section) that both $\mathbf{3 b}$ and $\mathbf{3 a}$ are always present in the samples. 


\section{Conclusions and perspectives}

To conclude, through a combined theoretical and experimental investigation of a series of yttrium-based complexes containing NIT radicals, we show that through-bond and throughspace NIT-NIT interactions can both be effective as soon as the inter-molecular NIT $\cdots$ NIT distance remains smaller than 4-5 $\AA$. This second interaction pathway strongly questions the up-to-now systematic use of the Bleaney-Bowers model to analyse the magnetic interactions in such complexes through fitting of the experimental data. Indeed, even if this model leads to a good quality fit, it does not give a realistic physical picture of the magnetic system. In this, state-of-the-art ab initio calculations, even if not fully quantitative, are the best tools to avoid loss of information but also potential over-parametrization.

Thanks to orbital localization procedures, we elucidate the microscopic mechanisms behind the through-bond NIT-NIT interaction, for which a predominant role is played by the $\pi$ orbitals of the $\mathrm{hfac}^{-}$ligand orthogonal to the $\mathrm{O}_{1}-\mathrm{O}_{2}$ direction. Though, one should keep in mind that this mechanism might not hold similarly for the isostructural lanthanide derivatives of the $\mathrm{Y}(\mathrm{hfac})_{3}(\mathrm{NIT}-\mathrm{R})_{2}$ compounds studied here. Indeed, in other trivalent lanthanide ions, contrary to the Y(III) ion and even if shielded by the outer shells, the $4 \mathrm{f}$ orbitals are not empty and could be involved in the NIT-NIT interaction mechanisms, maybe through spin-polarization effects, as suggested by Benelli and Gatteschi, $\underline{46}$ but also through the empty 6s, $6 \mathrm{p}$ and $5 \mathrm{~d}$ shells which have a larger radical expansion than the $4 \mathrm{f}$ shell. To support this hypothesis of non-transferability, one could refer to the work by Sutter and Kahn. ${ }^{92}$ Using the HDVV Hamiltonian, they extract the coupling constant in isostructural Y(III), La(III) and $\mathrm{Lu}(\mathrm{III})$ complexes, which are all diamagnetic but with different electronic structures (empty $4 \mathrm{f}$ shell in Y(III) and La(III) while completely filled in Lu(III)). Surprisingly, the coupling constants are not identical for all complexes, suggesting different operating mechanisms. In addition, contrary to the mechanism evidenced here, most of the mechanisms

discussed in the litterature for $\operatorname{Ln}(\mathrm{hfac})_{3}(\mathrm{NITR})_{2}$ complexes involve either the half-filled $4 \mathrm{f}$ orbitals or the virtual $5 \mathrm{~d}$ and $6 \mathrm{~s}$ orbitals of the lanthanide ion. $\frac{25|27| 34|43| 46|47| 92 \mid 95}{\text { To get a }}$ 
clearer picture of the through-bond NIT-NIT interaction in these lanthanide derivatives, and clarify whether or not the lanthanide orbitals are involved in the exchange mechanism, we are currently investigating the $\mathrm{Gd}(\mathrm{III})$ analogue of $\mathbf{1}$. Gd(III) is indeed a pure spin center and is thus expected to interact isotropically with the NIT radical ligands coordinated to it. This means that both the NIT-NIT and the Gd-NIT interactions can be described using the HDVV spin Hamiltonian.

\section{Acknowledgement}

C.J.C. acknowledges the financial support provided by the Ministerio de Economía y Competitividad (Spain) and FEDER funds through the project CTQ-2015-69019-P and the technical support of the Supercomputing Team of the Centro Informático Científico de Andalucía (CICA). J.J. thanks Rennes Metropole for financial support. Pr R. Sessoli, Pr A. Caneschi, Dr. C. Sangregorio and Dr L. Bogani are acknowledged for discussion. 


\section{References}

1. Kahn, O. Molecular Magnetism; VCH, 1993.

2. Sessoli, R.; Gatteschi, D.; Caneschi, A.; Novak, M. A. Magnetic bistability in a metalion cluster. Nature 1993, 365, 141-143.

3. Gatteschi, D.; Sessoli, R.; Villain, J. Molecular Nanomagnets; Oxford University Press, 2006.

4. Leuenberger, M. N.; Loss, D. Quantum computing in molecular magnets. Nature 2001, 410, 789-793.

5. Sanvito, S. Molecular spintronics. Chem. Soc. Rev. 2011, 40, 3336-3355.

6. Bogani, L.; Wernsdorfer, W. Molecular spintronics using single-molecule magnets. Nature Materials 2008, \%, 179-186.

7. Candini, A.; Klyatskaya, S.; Ruben, M.; Wernsdorfer, W.; Affronte, M. Graphene spintronic devices with molecular nanomagnets. Nano Lett. 2011, 11, 2634-2639.

8. Ferrando-Soria, J.; Moreno Pineda, E.; Chiesa, A.; Fernandez, A.; Magee, S. A.; Carretta, S.; Santini, P.; Vitorica-Yrezabal, I. J.; Tuna, F.; Timco, G. A. et al. A modular design of molecular qubits to implement universal quantum gates. Nature Commun. 2016, ґ, 11377.

9. Liu, J.-L.; Chen, Y.-C.; Guo, F.-S.; Tong, M.-L. Recent advances in the design of magnetic molecules for use as cryogenic magnetic coolants. Coord. Chem. Rev. 2014, 281, 26-49.

10. Christou, G.; Gatteschi, D.; Hendrickson, D. N.; Sessoli, R. Single-Molecule Magnets. MRS Bull. 2000, 25, 66-71. 
11. Gatteschi, G.; Sessoli, R. Quantum tunneling of magnetization and related phenomena in molecular materials. Angew. Chem. Int. Ed. 2003, 42, 268-297.

12. Bar, A. K.; Pichon, C.; Sutter, J.-P. Magnetic anisotropy in two- to eight-coordinated transition-metal complexes: Recent developments in molecular magnetism. Coord. Chem. Rev. 2016, 308, $346-380$.

13. Waldmann, O. A criterion for the anisotropy barrier in single-molecule magnets. Inorg. Chem. 2007, 46, 10035-10037.

14. Pointillart, F.; Bernot, K.; Sessoli, R.; Gatteschi, D. Effects of 3d-4f magnetic exchange interactions on the dynamics of the magnetization of $\mathrm{Dy}^{I I I}-\mathrm{M}^{I I}-\mathrm{Dy}{ }^{I I I}$ trinuclear clusters. Chem. Eur. J. 2007, 13, 1602-1609.

15. Gysler, M.; El Hallak, F.; Ungur, L.; Marx, R.; Hakl, M.; Neugebauer, P.; Rechkemmer, Y.; Lan, Y.; Sheikin, I.; Orlita, M. et al. Multitechnique investigation of Dy ${ }_{3}^{-}$ implications for coupled lanthanide clusters. Chem. Sci. 2016, 7, 4347-4354.

16. Layfield, R. A. Organometallic single-molecule magnets. Organometallics 2014, 33, 1084-1099.

17. Woodruff, D. N.; Winpenny, R. E. P.; Layfield, R. A. Lanthanide single-molecule magnets. Chem. Rev. 2013, 113, 5110-5148.

18. Luis, F.; Martínez-Pérez, M. J.; Montero, O.; Coronado, E.; Cardona-Serra, S.; MartíGastaldo, C.; Clemente-Juan, J. M.; Sesé, J.; Drung, D.; Schurig, T. Spin-lattice relaxation via quantum tunneling in an $\mathrm{Er}^{3+}$-polyoxometalate molecular magnet. Phys. Rev. B 2010, 82, 060403.

19. Pointillart, F.; Bernot, K.; Golhen, S.; Le Guennic, B.; Guizouarn, T.; Ouahab, L.; Cador, O. Magnetic memory in an isotopically enriched and magnetically isolated mononuclear dysprosium complex. Angew. Chem. Int. Ed. 2015, 54, 1504-1507. 
20. Demir, S.; Jeon, 1.-R.; Long, J. R.; Harris, T. D. Radical ligand-containing singlemolecules magnets. Coord. Chem. Rev. 2015, 289-290, 149-176.

21. Sessoli, R.; Bernot, K. In Lanthanides and actinides in molecular magnetism; Layfield, R. A., Murugesu, M., Eds.; Wiley-VCH, 2015; pp 89-124.

22. Rinehart, J. D.; Fang, M.; Evans, W. J.; Long, J. R. Strong exchange and magnetic blocking in $\mathrm{N}_{2}^{3-}$-radical-bridged lanthanide complexes. Nature Chem. 2011, 3, 538-542.

23. Rinehart, J. D.; Fang, M.; Evans, W. J.; Long, J. R. A $\mathrm{N}_{2}^{3-}$ radical-bridged terbium complex exhibiting magnetic hysteresis at 14 K. J. Am. Chem. Soc. 2011, 133, 1423614239 .

24. Meihaus, K. R.; Cordey, J. F.; Fang, M.; Ziller, J. W.; Long, J. R.; Evans, W. J. Influence of an Inner-Sphere $\mathrm{K}^{+}$Ion on the Magnetic Behavior of $\mathrm{N}_{2}^{3-}$ Radical-Bridged Dilanthanide Complexes Isolated Using an External Magnetic Field. Inorg. Chem. 2014, 53, 3099-3107.

25. Benelli, C.; Caneschi, A.; Gatteschi, D.; Pardi, L.; Rey, P.; Shum, D. P.; Carlin, R. L. Magnetic properties of lanthanide complexes with nitronyl nitroxides. Inorg. Chem. 1989, 28, 272-275.

26. Bogani, L. A perspective on slow-relaxing molecular magnets built from rare-earths and nitronyl-nitroxide building blocks. J. Appl. Phys. 2011, 109, $07 \mathrm{~B} 115$.

27. Benelli, C.; Gatteschi, D. Magnetism of lanthanides in molecular materials with transition-metal ion and organic radicals. Chem. Rev. 2002, 102, 2369-2387.

28. Benelli, C.; Gatteschi, D. Introduction to molecular magnetism: from transition metals to lanthanides; Wiley-VCH, 2015. 
29. Gupta, T.; Rajeshkumar, T.; Rajaraman, G. Magnetic exchange in Gd $^{I I I}$-radical complexes: method assessment, mechanism of coupling and magneto-structural correlations. Phys. Chem. Chem. Phys. 2014, 16, 14568-14577.

30. Zhu, M.; Li, C.; Wang, X.; Li, L.; Sutter, J.-P. Thermal magnetic hysteresis in a copper-gadolinium-radical chain compound. Inorg. Chem. 2016, 55, 2676-2678.

31. Zhu, M.; Mei, X.; Ma, Y.; Li, L.; Liao, D.; Sutter, J.-P. Nitronyl nitroxide-metal complexes as metallo-ligands for the construction of hetero-tri-spin (2p-3d-4f) chains. Chem. Commun. 2014, 50, 1906-1908.

32. Zhu, M.; Wang, J.; Yang, M.; Ma, Y.; Li, L. Slow magnetic relaxation in twodimensional 3d-4f complexes based on phenyl pyrimidyl substituted nitronyl nitroxide radicals. Dalton Trans. 2015, 44, 9815-9822.

33. Wang, X.-F.; Hu, P.; Li, Y.-G.; Li, L.-C. Construction of nitronyl nitroxide-based 3d-4f clusters: structure and magnetism. Chem. Asian J. 2015, 10, 325-328.

34. Benelli, C.; Caneschi, A.; Gatteschi, D.; Pardi, L.; Rey, P. Linear-chain gadolinium(III) nitronyl nitroxide complexes with dominant next-nearest-neighbor magnetic interactions. Inorg. Chem. 1990, 29, 4223-4228.

35. Lascialfari, A.; Ullu, R.; Affronte, M.; Cinti, F.; Caneschi, A.; Gatteschi, D.; Rovai, D.; Pini, M.; Rettori, A. Indication for a chiral phase in the molecular magnetic chain Gd(hfac) ${ }_{3} \mathrm{NiTiPr}$ by specific heat and $\mu^{+} \mathrm{SR}$ measurements. J. Magn. Magn. Mater. 2004, 272-276, Part 2, 1052-1053.

36. Cinti, F.; Rettori, A.; Barucci, M.; Olivieri, E.; Risegari, L.; Ventura, G.; Caneschi, A.; Gatteschi, D.; Rovai, D.; Pini, M. et al. Evidence for a helical and a chiral phase transition in the magnetic specific heat. J. Magn. Magn. Mater. 2007, 310, 1460-1461. 
37. Cinti, F.; Rettori, A.; Pini, M.; Mariani, M.; Micotti, E.; Lascialfari, A.; Papinutto, N.; Amato, A.; Caneschi, A.; Gatteschi, D. et al. Experimental validation of Villain's conjecture about magnetic ordering in quasi-1D helimagnets. J. Magn. Magn. Mater. 2010, 322, 1259-1261.

38. Bogani, L.; Sangregorio, C.; Sessoli, R.; Gatteschi, D. Molecular engineering for singlechain-magnet behavior in a one-dimensional dysprosium-nitronyl nitroxide compound. Angew. Chem. Int. Ed. 2005, 44, 5817-5821.

39. Bernot, K.; Bogani, L.; Caneschi, A.; Gatteschi, D.; Sessoli, R. A family of rare-earthbased single chain magnets: playing with anisotropy. J. Am. Chem. Soc. 2006, 128, $7947-7956$.

40. Bernot, K.; Bogani, L.; Sessoli, R.; Gatteschi, D. [Tm $\left.{ }^{I I}(\text { hfac })_{3}(\mathrm{NITPhOPh})\right] \infty$ : a new member of a lanthanide-based single chain magnets family. Inorg. Chim. Acta 2007, 360, 3807-3812.

41. Bernot, K.; Luzon, J.; Caneschi, A.; Gatteschi, D.; Sessoli, R.; Bogani, L.; Vindigni, A.; Rettori, A.; Pini, M. G. Spin canting in a Dy-based single-chain magnet with dominant next-nearest-neighbor antiferromagnetic interactions. Phys. Rev. B 2009, 79, 134419.

42. Bernot, K.; Luzon, J.; Bogani, L.; Etienne, M.; Sangregorio, C.; Shanmugam, M.; Caneschi, A.; Sessoli, R.; Gatteschi, D. Magnetic anisotropy of dysprosium(III) in a lowsymmetry environment: a theoretical and experimental investigation. J. Am. Chem. Soc. 2009, 131, 5573-5579.

43. Benelli, C.; Caneschi, A.; Gatteschi, D.; Rey, P. Structure and magnetic properties of a gadolinium hexafluoroacetylacetonate adduct with the radical 4,4,5,5-tetramethyl-2phenyl-4,5,-dihydro-1H-imidazole 3-Oxide 1-Oxyl. Angew. Chem. Int. Ed. 1987, 26, $913-915$. 
44. Benelli, C.; Caneschi, A.; Gatteschi, D.; Pardi, L.; Rey, P. Structure and magnetic properties of linear-chain complexes of rare-earth ions (gadolinium, europium) with nitronyl nitroxides. Inorg. Chem. 1989, 28, 275-280.

45. Benelli, C.; Caneschi, A.; Gatteschi, D.; Pardi, L.; Rey, P. One-dimensional magnetism of a linear chain compound containing yttrium(III) and a nitronyl nitroxide radical. Inorg. Chem. 1989, 28, 3230-3234.

46. Benelli, C.; Caneschi, A.; Gatteschi, D.; Sessoli, R. Magnetic interactions and magnetic ordering in rare earth metal nitronyl nitroxide chains. Inorg. Chem. 1993, 32, 47974801.

47. Benelli, C.; Caneschi, A.; Gatteschi, D.; Pardi, L.; Rey, P. Magnetic properties and phase transitions in molecular based materials containing rare earth ions and organic radicals. J. Appl. Phys. 1993, 73, 5333-5337.

48. Champion, G.; Lalioti, N.; Tangoulis, V.; Arrio, M.-A.; Sainctavit, P.; Villain, F.; Caneschi, A.; Gatteschi, D.; Giorgetti, C.; Baudelet, F. et al. XMCD for monitoring exchange interactions. The role of the $\mathrm{Gd} 4 \mathrm{f}$ and $5 \mathrm{~d}$ orbitals in metal-nitronyl nitroxide magnetic chains. J. Am. Chem. Soc. 2003, 125, 8371-8376.

49. Launay, J.-P.; Verdaguer, M. Electrons in molecules: from basic principles to molecular electronics; Oxford University Press, 2014.

50. Bolvin, H. An alternative approach to the g-matrix: theory and applications. Chem. Phys. Chem. 2006, 7, 1575-1589.

51. Chibotaru, L. F.; Ceulemans, A.; Bolvin, H. Unique definition of the Zeeman-splitting g tensor of a Kramers doublet. Phys. Rev. Lett. 2008, 101, 033003.

52. Chibotaru, L. F.; Ungur, L.; Soncini, A. The origin of nonmagnetic Kramers doublets 
in the ground state of dysprosium triangles: evidence for a toroidal magnetic moment. Angew. Chem. Int. Ed. 2008, 47, 4126-4129.

53. Chibotaru, L. F.; Ungur, L. Ab initio calculation of anisotropic magnetic properties of complexes. I. Unique definition of pseudospin Hamiltonians an their derivation. $J$. Chem. Phys. 2012, 137, 064112.

54. Atanasov, M.; Aravena, D.; Suturina, E.; Bill, E.; Maganas, D.; Neese, F. First principles approach to the electronic structure, magnetic anisotropy and spin relaxation in mononuclear 3d-transition metal single molecule magnets. Coord. Chem. Rev. 2015, 289-290, 177-214.

55. Malrieu, J.-P.; Caballol, R.; Calzado, C. J.; de Graaf, C.; Guihéry, N. Magnetic interactions in molecules and highly correlated materials: physical content, analytical derivation, and rigorous extraction of magnetic Hamiltonians. Chem. Rev. 2014, 114, 429-492.

56. Maurice, R.; Graaf, C. d.; Guihery, N. Theoretical determination of spin Hamiltonians with isotropic and anisotropic magnetic interactions in transition metal and lanthanide complexes. Phys. Chem. Chem. Phys. 2013, 15, 18784-18804.

57. Ruamps, R.; Maurice, R.; de Graaf, C.; Guihéry, N. Interplay between local anisotropies in binuclear complexes. Inorg. Chem. 2014, 53, 4508-4516.

58. Iwahara, N.; Chibotaru, L. F. Exchange interaction between J multiplets. Phys. Rev. B 2015, 91, 174438.

59. Iwahara, N.; Chibotaru, L. F. New mechanism of kinetic exchange interaction induced by strong magnetic anisotropy. Scientific Reports 2016, 6, 24743.

60. Zheludev, A.; Barone, V.; Bonnet, M.; Delley, B.; Grand, A.; Ressouche, E.; Rey, P.; Subra, R.; Schweizer, J. Spin density in a nitronyl nitroxide free radical. Polarized 
neutron diffraction investigation and ab initio calculations. J. Am. Chem. Soc. 1994, 116, 2019-2027.

61. Ressouche, E.; Boucherle, J.-X.; Gillon, B.; Rey, P.; Schweizer, J. Spin density maps in nitroxide-copper(II) complexes. A polarized neutron diffraction determination. J. Am. Chem. Soc. 1993, 115, 3610-3617.

62. Angeli, C.; Calzado, C. J.; de Graaf, C.; Caballol, R. The electronic structure of Ullman's biradicals: an orthogonal valence bond interpretation. Phys. Chem. Chem. Phys. 2011, 13, 14617.

63. Zhao, Q.-H.; Ma, Y.-P.; Du, L.; Fang, R.-B. Three novel rare-earth complexes with nitronyl nitroxide radical $\left[\mathrm{RE}(\mathrm{hfac})_{3}\left(\mathrm{NITPhOCH}_{3}\right)_{2}\right]\left[\mathrm{RE}=\mathrm{Gd}^{I I I}, \mathrm{Y}^{I I I}\right.$ and $\left.\mathrm{Er}^{I I I}\right]$ : synthesis, crystal structures and magnetic properties. Trans. Met. Chem. 2006, 31, $593-597$.

64. Sheldrick, G. M. A short history of SHELX. Acta Crystallographica Section A 2008, $64,112-122$.

65. Altomare, A.; Burla, M. C.; Camalli, M.; Carrozzini, B.; Cascarano, G. L.; Giacovazzo, C.; Guagliardi, A.; Moliterni, A. G. G.; Polidori, G.; Rizzi, R. EXPO: a program for full powder pattern decomposition and crystal structure solution. Journal of Applied Crystallography 1999, 32, 339-340.

66. Sheldrick, G. M.; Schneider, T. R. SHELXL : High-Resolution Refinement. Macromolecular Crystallography Part B 1997, 319-343.

67. Farrugia, L. J. WinGX and ORTEP for Windows: an update. Journal of Applied Crystallography 2012, 45, 849-854.

68. Miralles, J.; Daudey, J.-P.; Caballol, R. Variational calculation of small energy differences. The singlet-triplet gap in $\left[\mathrm{Cu}_{2} \mathrm{Cl}_{6}\right]^{2-}$. Chem. Phys. Lett. 1992, 198, 555-562. 
69. Miralles, J.; Castell, O.; Caballol, R.; Malrieu, J.-P. Specific CI calculation of energy differences: Transition energies and bond energies. Chem. Phys. 1993, 172, 33-43.

70. CASDI package developed at the Laboratoire de Chimie et Physique Quantiques, Université Toulouse III - Paul Sabatier, Toulouse, France.

71. Ben Amor, N.; Maynau, D. Size-consistent self-consistent configuration interaction from a complete active space. Chem. Phys. Lett. 1998, 286, 211.

72. Aquilante, F.; De Vico, L.; Ferré, N.; Ghigo, G.; Malmqvist, P.-Å.; Neogrady, P.; Pedersen, T. B.; Pitoňák, M.; Reiher, M.; Roos, B. O. et al. MOLCAS 7: The next generation. J. Comput. Chem. 2010, 31, 224.

73. Bories, B.; Maynau, D.; Bonnet, M.-L. Selected excitation for CAS-SDCI calculations. J. Comput. Chem. 2007, 28, 632-643.

74. Roos, B. O.; Lindh, R.; Malmqvist, P.-A.; Veryazov, V.; Widmark, P.-O. Main group atoms and dimers studied with a new relativistic ANO basis set. J. Phys. Chem. A 2004, 108, 2851-2858.

75. Roos, B. O.; Lindh, R.; Malmqvist, P.-A.; Veryazov, V.; Widmark, P.-O. New relativistic ANO basis sets for transition metal atoms. J. Phys. Chem. A 2005, 109, 6575-6579.

76. Roos, B. O.; Lindh, R.; Malmqvist, P.-A.; Veryazov, V.; Widmark, P.-O.; Borin, A. C. New relativistic atomic natural orbital basis sets for lanthanide atoms with applications to the Ce diatom and $\mathrm{LuF}_{3}$. J. Phys. Chem. A 2008, 112, 11431-11435.

77. Hay, P. J.; Thibeault, J. C.; Hoffmann, R. Orbital interactions in metal dimer complexes. J. Am. Chem. Soc. 1975, 97, 4884-4899.

78. Anderson, P. W. Antiferromagnetism. Theory of superexchange interaction. Phys. Rev. 1950, 79, 350. 
79. Anderson, P. W. New approach to the theory of superexchange interactions. Phys. Rev. $1959,115,2-13$.

80. Kahn, O.; Briat, B. Exchange interaction in polynuclear complexes. Part 1.-Principles, model and application to the binuclear complexes of chromium(III). J. Chem. Soc., Faraday Trans. 2 1976, 72, 268-281.

81. Anderson, P. W. In Solid state physics: advances in research and applications; Turnbull, F., Seitz, F., Eds.; Academic Press: New York, 1963; p 99.

82. Hu, P.; Zhang, C.; Gao, Y.; Li, Y.; Ma, Y.; Li, L.; Liao, D. Lanthanide-radical linear chain compounds based on 2,4,4,5,5-pentamethylimidazoline-1-oxyl-3-oxide: Structure and magnetic properties. Inorg. Chim. Acta 2013, 389, 136-140.

83. Calzado, C. J.; Angeli, C.; de Graaf, C.; Caballol, R. Analysis of the magnetic coupling in nitroxide organic biradicals. Theor. Chem. Acc. 2011, 128, 505.

84. Novoa, J. J.; Lafuente, P.; Deumal, M.; Mota, F. In Magnetism: molecules to materials IV; Miller, J. S., Drillon, M., Eds.; Wiley-VCH: Weinheim, 2003; Chapter 3, p 65.

85. Suaud, N.; Ruamps, R.; Guihéry, N.; Malrieu, J.-P. A strategy to determine appropriate active orbitals and accurate magnetic couplings in organic magnetic systems. J. Chem. Theory Comput. 2012, 8, 4127-4137.

86. Novoa, J. J.; Deumal, M.; Jornet-Somoza, J. Calculation of microscopic exchange interactions and modelling of macroscopic magnetic properties in molecule-based magnets. Chem. Soc. Rev. 2011, 40, 3182-3212.

87. Angeli, C.; Calzado, C. J. The role of the magnetic orbitals in the calculation of the magnetic coupling constants from multireference perturbation theory methods. J. Chem. Phys. 2012, 137, 034104. 
88. Giner, E.; Angeli, C. Metal-ligand delocalization and spin density in the $\mathrm{CuCl}_{2}$ and $\left[\mathrm{CuCl}_{4}\right]^{2-}$ molecules: Some insights from wave function theory. J. Chem. Phys. 2015, $124305,143$.

89. Cabrero, J.; Calzado, C. J.; Maynau, D.; Caballol, R.; Malrieu, J.-P. Metal-ligand delocalization in magnetic orbitals of binuclear complexes. J. Phys. Chem. A 2002, $106,8146$.

90. Garcia, V. M.; Castell, O.; Caballol, R.; Malrieu, J.-P. An iterative difference-dedicated configuration interaction. Proposal and test studies. Chem. Phys. Lett. 1995, 238, 222229 .

91. Spivak, M.; Angeli, C.; Calzado, C.; de Graaf, C. Improving the calculation of magnetic coupling constants in MRPT methods. J. Comp. Chem. 2014, 35, 1665-1671.

92. Kahn, M. L.; Sutter, J.-P.; Gohlen, S.; Guinneau, P.; Ouahab, L.; Kahn, O.; Chasseau, D. Systematic investigation of the nature of the coupling between a $\operatorname{Ln}($ III) ion ( $\operatorname{Ln}$ $=\mathrm{Ce}(\mathrm{III})$ to $\mathrm{Dy}(\mathrm{III}))$ and its aminoxyl radical ligands. Structural and magnetic characteristics of a series of $\operatorname{Ln}(\text { organic radical })_{2}$ compounds and the related $\operatorname{Ln}(\text { nitrone })_{2}$ derivatives. J. Am. Chem. Soc. 2000, 122, 3413-3421.

93. Kahn, M. L.; Ballou, R.; Porcher, P.; Kahn, O.; Sutter, J.-P. Analytical determination of the Ln-aminoxyl radical exchange interaction taking into account both the ligandfield effect and the spin-orbit coupling of the lanthanide ion $\left(\mathrm{Ln}=\mathrm{Dy}^{I I I}\right.$ and $\left.\mathrm{Ho}^{I I I}\right)$. Chem. Eur. J. 2002, 8, 525-531.

94. Sutter, J.-P.; Kahn, M. L.; Gohlen, S.; Ouahab, L.; Kahn, O. Synthesis and magnetic behavior of rare-earth complexes with N,O-chelating nitronyl nitroxide triazole ligands: example of a $\left[\mathrm{Gd}^{I I I}\right.$ organic radical 2$]$ compound with an $\mathrm{S}=9 / 2$ ground state. Chem. Eur. J. 1998, 4, 571-576. 
95. Sutter, J.-P.; Kahn, M. L.; Kahn, O. Conclusive demonstration of the ferromagnetic nature of the interaction between Holmium(III) and aminoxyl radicals. Adv. Mater. 1999, 11, 863-865.

96. Benelli, C.; Caneschi, A.; Fabretti, A. C.; Gatteschi, D.; Pardi, L. Ferromagnetic coupling of gadolinium(III) ions and nitronyl nitroxide radicals in an essentially isotropic way. Inorg. Chem. 1990, 29, 4153-4155.

97. Maynau, D.; Evangelisti, S.; Guihéry, N.; Calzado, C. J.; Malrieu, J.-P. Direct generation of local orbitals for multireference treatment and subsequent uses for the calculation of the correlation energy. J. Chem. Phys. 2002, 116, 10060.

98. Calzado, C. J.; Ben Amor, N.; Maynau, D. Magnetic coupling constants of selfassembled $\mathrm{Cu}(\mathrm{II})$ [3x3] grids: alternative spin model from theoretical calculations. Chem. Eur. J. 2014, 20, 8979-8987.

99. Calzado, C. J. On the controversial fitting of susceptibility curves of ferromagnetic $\mathrm{Cu}$ (II) cubanes: insights from theoretical calculations. Chem. Eur. J. 2013, 19, 12541261.

100. Chang, C.; Calzado, C. J.; Ben Amor, N.; Sánchez-Marín, J.; Maynau, D. Multi-scale multireference configuration interaction calculations for large systems using localized orbitals: Partition in zones. J. Chem. Phys. 2012, 137, 104102.

101. Tenti, L.; Maynau, D.; Angeli, C.; Calzado, C. J. Highly efficient perturbative plus variational strategy based on orthogonal valence bond theory for the evaluation of magnetic coupling constants. Application to the trinuclear $\mathrm{Cu}(\mathrm{II})$ site of multicopper oxidases. Phys. Chem. Chem. Phys. 2016, 18, 18365-18380.

102. Calzado, C. J.; Cabrero, J.; Malrieu, J. P.; Caballol, R. Analysis of the magnetic coupling in binuclear complexes. I. Physics of the coupling. J. Chem. Phys. 2002, 116, $2728-2747$. 
103. Calzado, C. J.; Cabrero, J.; Malrieu, J. P.; Caballol, R. Analysis of the magnetic coupling in binuclear complexes. II. Derivation of valence effective Hamiltonians from an initio $\mathrm{Cl}$ and DFT calculations. J. Chem. Phys. 2002, 116, 3985-4000.

104. Calzado, C. J.; Angeli, C.; Taratiel, D.; Caballol, R.; Malrieu, J.-P. Analysis of the magnetic coupling in binuclear complexes. III. The role of the ligand to metal charge transfer excitations revisited. J. Chem. Phys. 2009, 131, 044327.

105. Rota, J.-B.; Calzado, C. J.; Train, C.; Robert, V. Microscopic origins of the ferromagnetic exchange coupling in oxoverdazyl-based $\mathrm{Cu}(\mathrm{II})$ complex. J. Chem. Phys. 2010, 132, 154702.

106. Calzado, C. J.; Angeli, C.; Caballol, R.; Malrieu, J.-P. Extending the active space in multireference configuration interaction calculations of magnetic coupling constants. Theor. Chem. Acc. 2010, 126, 185-196.

107. Borras-Almenar, J. J.; Coronado, E.; Curely, J.; Georges, R.; Gianduzzo, J. C. Alternating chains with ferromagnetic and antiferromagnetic interactions. Theory and magnetic properties. Inorg. Chem. 1994, 33, 5171-5175. 


\section{Graphical TOC Entry}

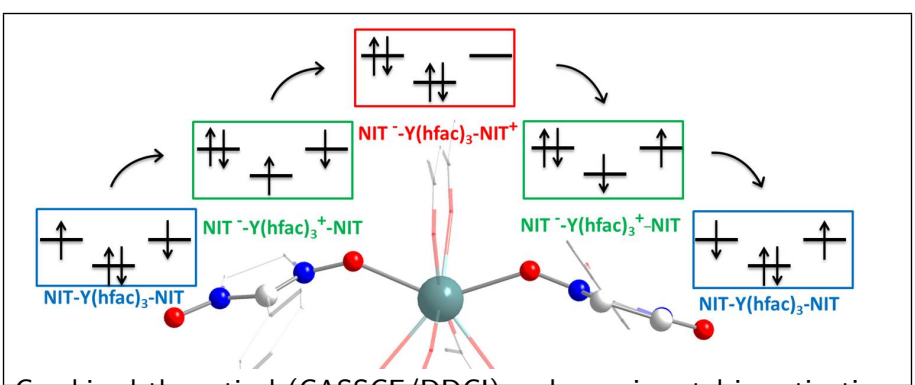

Combined theoretical (CASSCF/DDCI) and experimental investigations of a series of yttrium-based complexes containing nitronyl-nitroxide radicals (NIT) demonstrate that through-bond and through-space interactions can be effective for intermolecular distances as high as $5 \AA$. Orbital localization procedure highlights that $\pi$ orbitals of hfac ${ }^{-}$ligands play a predominant role in mediating the through-bond NIT-NIT interaction. 